University of Nebraska - Lincoln

DigitalCommons@University of Nebraska - Lincoln

$12-19-2002$

\title{
Two-Photon Bremsstrahlung Processes in Atoms: Polarization Effects and Analytic Results for the Coulomb Potential
}

\author{
A. A. KrylovetskiI \\ Voronezh State University \\ N. L. Manakov \\ Voronezh State University, manakov@phys.vsu.ru
}

S. I. Marmo

Voronezh State University

Anthony F. Starace

University of Nebraska-Lincoln, astarace1@unl.edu

Follow this and additional works at: https://digitalcommons.unl.edu/physicsstarace

Part of the Physics Commons

KrylovetskiĬ, A. A.; Manakov, N. L.; Marmo, S. I.; and Starace, Anthony F., "Two-Photon Bremsstrahlung Processes in Atoms: Polarization Effects and Analytic Results for the Coulomb Potential" (2002). Anthony F. Starace Publications. 118.

https://digitalcommons.unl.edu/physicsstarace/118

This Article is brought to you for free and open access by the Research Papers in Physics and Astronomy at DigitalCommons@University of Nebraska - Lincoln. It has been accepted for inclusion in Anthony F. Starace Publications by an authorized administrator of DigitalCommons@University of Nebraska - Lincoln. 


\title{
Two-Photon Bremsstrahlung Processes in Atoms: Polarization Effects and Analytic Results for the Coulomb Potential
}

\author{
A. A. Krylovetskiï ${ }^{a}$, N. L. Manakova, ${ }^{a}$, S. I. Marmo ${ }^{a}$, and A. F. Starace ${ }^{b}$ \\ ${ }^{a}$ Voronezh State University, Universitetskaya pl. 1, Voronezh, 394006 Russia \\ ${ }^{b}$ Department of Physics and Astronomy, University of Nebraska, Lincoln NE 68588-0111, USA \\ *e-mail:manakov@thp.vsu.ru \\ Received June 7, 2002
}

\begin{abstract}
The partial wave analysis of two-photon free-free (bremsstrahlung) electron transition cross sections during scattering by a static potential $U(r)$, as well as by an atom with a nonzero angular momentum, is carried out. The dipole interaction with radiation is taken into account in the second order of perturbation theory for the general case of elliptic polarization of photons. The polarization and angular dependences of the twophoton potential scattering amplitude is presented as a combination of the scalar product of electron momenta and photon polarization vectors and five atomic parameters containing Legendre polynomials of the scattering angle as well as radial matrix elements depending on the initial $(E)$ and final $\left(E^{\prime}\right)$ electron energies. The results are applicable both for spontaneous double bremsstrahlung at nonrelativistic energies and for induced absorption and emission in the field of a light wave. Specific polarization effects (circular and elliptic dichroism) are analyzed for two-photon bremsstrahlung processes associated with the interference of the Hermite and antiHermite parts of the amplitude and depending on the sign of photon helicity. The limiting cases of high and low photon frequencies are investigated analytically, and the asymptotic forms of radial matrix elements and amplitudes for the general form of the $U(r)$ potential are determined. Closed analytic expressions are derived for the radial matrix elements of the Coulomb potential in the form of integrals of hypergeometric function, and singularities are singled out in explicit form for $E^{\prime} \longrightarrow E$. The methods of approximate calculation of the radial matrix elements are discussed, and the results of their exact numerical calculation, as well as angular distributions and the cross sections of induced one- and two-photon emission and absorption, are given for the case of the Coulomb potential. The numerical results show that dichroism effects are quite accessible for experimental observations. (C) 2002 MAIK "Nauka/Interperiodica".
\end{abstract}

\section{INTRODUCTION}

Electron scattering by atoms and ions accompanied by emission and absorption of photons constitutes a significant branch in atomic physics. A quantummechanical description of such processes originates from the experiments on spontaneous bremsstrahlung (BrS) carried out in 1931 by Sommerfeld, who studied electron scattering by a Coulomb center [1]. In the nonrelativistic dipole approximation, the $\mathrm{BrS}$ cross section with the emission of a photon of frequency $\omega$ and polarization vector $\mathbf{e}$ in direction $\mathbf{k}$,

$$
\frac{d \sigma}{d \omega d \Omega_{\mathbf{p}^{\prime}} d \Omega_{\mathbf{k}}}=\frac{e^{2} \hbar^{5}}{(2 \pi)^{4} c^{3}} \frac{p^{\prime}}{p} \omega|\mathcal{M}|^{2}
$$

is determined by the matrix element (ME)

$$
\mathcal{M}=\left\langle\psi_{\mathbf{p}^{\prime}}^{(-)}\left|\mathbf{e}^{*} \cdot \nabla\right| \psi_{\mathbf{p}}^{(+)}\right\rangle
$$

of the transition between the states $\psi_{\mathbf{p}}^{(+)}$and $\psi_{\mathbf{p}^{\prime}}^{(-)}$of the continuous electron spectrum in the static atomic potential $U(r)$. For scattering by a Coulomb center, the 3D ME $M$ can be calculated in terms of hypergeometric functions ${ }_{2} F_{1}(a, b ; c ; x)[1,2]$. Moreover, it is possible in this case to analytically integrate cross section (1) over the directions of scattered electron and to express the $\mathrm{BrS}$ spectral distribution $d \sigma / d \omega$ in closed form in terms of the derivative of the squared modulus of function ${ }_{2} F_{1}$ with respect to the argument (Sommerfeld formula $[1,3])$. The calculation of cross section (1) for the general form of potential $U(r)$ is based on the use of multipole expansion of function $\psi_{\mathbf{p}}^{( \pm)}$(see formula (15) below). In this case, the partial expansion of amplitude $\mathcal{M}$, which is convenient for analysis of the polarization-angular dependence of the cross section, has the form [4]

$$
\begin{gathered}
M=Q\left(p, p^{\prime}, \theta\right)\left(\mathbf{e}^{*} \cdot \mathbf{p}\right)+Q\left(p^{\prime}, p, \theta\right)\left(\mathbf{e}^{*} \cdot \mathbf{p}^{\prime}\right), \\
Q\left(p, p^{\prime}, \theta\right)=i \frac{2 \pi^{2}}{m \sqrt{p^{3} p^{\prime}}} \sum_{l=1}^{\infty}\left[\exp \left(i \Delta_{l-}\right) d_{l-1}\left(E^{\prime}, E\right)\right. \\
\left.+\exp \left(i \Delta_{l+}\right) d_{l+1}\left(E^{\prime}, E\right)\right] P_{l}^{(1)}(\cos \theta) .
\end{gathered}
$$

Here, $\Delta_{l \pm}=\delta_{l \pm 1}\left(p^{\prime}\right)+\delta_{l}(p), \delta_{l}(p)$ are the phases of scattering at potential $U(r), P_{l}^{(1)}(x)=(d / d x) P_{l}(x)$ is the derivative of the Legendre polynomial $P_{l}(x), E^{\prime}=$ $p^{\prime 2} / 2 m=E-\hbar \omega$, and $\delta_{l^{\prime}, l}\left(E^{\prime}, E\right)$ are the radial MEs of 
the momentum operator (see formula (37)). The spectral distribution $d \sigma / d \omega$ can also be written in the form of a partial series:

$$
\begin{gathered}
\frac{d \sigma}{d \omega}=\frac{8 \pi^{2} e^{2} \hbar^{5}}{3 m^{2} c^{3}} \frac{\omega}{p^{2}} \\
\times \sum_{l=1}^{\infty} l\left[\left|d_{l l-1}\left(E^{\prime}, E\right)\right|^{2}+\left|d_{l-1 l}\left(E^{\prime}, E\right)\right|^{2}\right] .
\end{gathered}
$$

In the case of a Coulomb potential, this series can be summed directly (see [5], where the sum of series (5) written using the interaction operator in the "form of acceleration" is calculated analytically) and the Sommerfeld formula can be reproduced. Although such an approach is of rather methodical interest for Coulombtype $\mathrm{BrS}$, the partial wave analysis for a $U(r)$ potential of the general form is the only method of simplifying general formulas (1) and (2) without using additional approximations.

Apart from conventional $\mathrm{BrS}$, the scattering of an electron at a force center can be accompanied by simultaneous emission of two spontaneous photons (double bremsstrahlung, 2BrS), which was considered for the first time in the general form by Heitler and Nordheim in 1934 [6] as a radiation correction to conventional BrS. In 1985, spontaneous $2 \mathrm{BrS}$ was detected experimentally (see [7] and subsequent publications [8], where the differential cross sections of emission of two bremsstrahlung photons during scattering of electrons with an energy of about $70 \mathrm{keV}$ by thin targets were measured by the coincidence method). In experiments [9], spontaneous $2 \mathrm{BrS}$ was observed for electrons with energy of the order of $10 \mathrm{keV}$. The first theoretical calculations of the $2 \mathrm{BrS}$ cross section for electron scattering by a nucleus were made in the framework of the relativistic Born approximation [10]. The action of the Coulomb field on the electron in a $2 \mathrm{BrS}$ process can be taken into account exactly in the nonrelativistic dipole approximation. Using the Coulomb Green function, the $2 \mathrm{BrS}$ amplitude can be presented in the form of integrals of the hypergeometric function ${ }_{2} F_{1}$ (a two-photon analog of the results obtained in [1,2] for $M$ in Eq. (2)). Different methods of calculation of two-photon amplitudes (with different representations of the Coulomb Green function) used by different authors lead to quantitatively equivalent expressions, but having different forms [11-13]. In particular, the integrated ("Born") terms were singled out in the amplitude in [13], which considerably simplifies analysis of the limiting cases. It is worth mentioning the effective approximate method of calculation of the $2 \mathrm{BrS}$ amplitude proposed by Korol [14] by taking into account only the contribution for $\delta$ shaped singularities emerging for $E_{2} \longrightarrow E_{1}$ in the single-photon MMs $d_{l_{2} l_{1}}\left(E_{2}, E_{1}\right)$ appearing in the compound ME of the two-photon transition. This method was subsequently extended to nondipole calculations [15] and to the relativistic case [16]. Exact analytic expressions for the nonrelativistic $2 \mathrm{BrS}$ amplitude taking into account delay effects were obtained in $[17,18]$. In addition to the above results obtained for the Coulomb potential, numerical calculations of spontaneous $2 \mathrm{BrS}$ were also made for electron scattering by neutral atoms both in the model of potential scattering [19] and in the model taking into account the polarization bremsstrahlung emitted by an atomic core [20].

In addition to spontaneous emission, considerable interest in multiquanta bremsstrahlung processes was stimulated by laser experiments, which made it possible to observe induced multiphoton bremsstrahlung emission and absorption in the optical frequency range. The first measurements of the cross sections of freefree electron transitions in the presence of a high-intensity laser wave were made in $[21,22]$. Such experiments were subsequently repeated more than once with different atomic targets for different electron beam energies and experimental geometries (see, for example, [23] and review [24]). Quite general results could be obtained in the theoretical description of multiphoton transitions in the continuous spectrum in the Born and low-frequency approximations. In the Born case, the cross section $d \sigma^{n}$ of the $n$-photon induced emission $(n<0)$ and absorption $(n>0)$ in a laser field with amplitude $F$, polarization vector $\mathbf{e}$, and frequency $\omega$ has a simple form (the Bunkin-Fedorov formula [25]; see also [26]):

$$
d \sigma^{n}=\frac{p_{n}^{\prime}}{p} J_{n}^{2}\left(\frac{e F\left|\mathbf{e} \cdot\left(\mathbf{p}-\mathbf{p}_{n}^{\prime}\right)\right|}{m \hbar \omega^{2}}\right) d \sigma_{B},
$$

where $J_{n}$ is the Bessel function, $d \sigma_{B}$ is the Born elastic scattering cross section in the absence of a light wave, and momenta $p$ and $p_{n}^{\prime}$ in the initial and final states are associated with the energy conservation law: $\left(p_{n}^{\prime 2}-\right.$ $\left.p^{2}\right) / 2 m=n \hbar \omega$. It was shown in [27] that the Born series can be summed exactly in the low-frequency limit $(\omega \longrightarrow 0)$ so that cross section $d \sigma^{n}$ also has factorized form (6) in which $d \sigma_{B}$ is replaced by the exact elastic scattering cross section $d \sigma_{0}$ in zero light field even for slow electrons for which $\hbar \omega \ll E$. It should be noted that, although various versions were proposed for deriving the low-frequency asymptotic form (see, for example, $[28,29])$, the limits of applicability of the Kroll-Watson approximation [27] disregarding the action of the laser field on the dynamics of interaction between a slow electron and the atomic potential are still a subject of discussions [30-33]. In [34], the expression for $d \sigma^{n}$ was derived in the approximation in which the motion of an electron is described classically and the emission and absorption processes are described quantum-mechanically. Various versions of generalization of the results obtained in [25, 27] taking into account the effects of a strong laser field are given, for example, in review [35]; however, the scattering potential can be taken into account exactly only in per- 
turbation theory in the field of the wave. In particular, such calculations for the Coulomb-type double bremsstrahlung emission and absorption is completely identical to the case of spontaneous 2BrS [11-13]. However, a special situation emerges in the case of elastic reemission of photons: both MEs determining the transition amplitude are diverging; consequently, we must eliminate the divergence to obtain a finite result $[13,36]$. In addition to purely stimulated transitions induced by a high-intensity laser field, the latter may modify spontaneous BrS also. This question was investigated in [37] in the Born approximation. A more detailed inclusion of the effects of an atomic (Coulomb) potential was carried out in [38, 39]. It is also appropriate to mention publications [40, 41], in which "combined" Compton-type bremsstrahlung processes (absorption of a laser photon by an electron followed by spontaneous BrS in the field of the nucleus) were considered.

In view of the difficulties encountered in the experimental determination of the polarization characteristics of spontaneous BrS, the energy and angular dependences of cross sections have been mainly analyzed in publications devoted to spontaneous bremsstrahlung processes. On the contrary, in the case of induced processes, the possibility of controllable variation of laser polarization opens new prospects for studying the freefree transitions, which stimulates an analysis of polarization effects in bremsstrahlung processes. A generalization of the results obtained by Kroll and Watson [27] to the case of elliptic polarization of laser radiation is considered in $[31,42]$. A considerable difference in the one- and two-photon scattering cross sections in the cases of linear and circular laser polarizations for electrons scattered by hydrogen [43] and helium [44] atoms is demonstrated in $[43,44]$. However, the most impressive polarization effect is the dichroism manifested in the difference in the cross sections for the opposite polarities of the degree of circular polarization of photons. It was found [4] that the differential cross section of single-photon $\mathrm{BrS}$ for electron scattering by a nucleus differs significantly for photons with right and left circular polarizations (circular dichroism, CD). A general analysis of the $\mathrm{CD}$ in bremsstrahlung in the case when an electron is scattered by an atom with a nonzero angular momentum was carried out in [45]. The polarization dependence of corrections to the Coulomb scattering, which are associated with the effect of the light wave, was studied in $[13,36]$. The CD effect is sensitive to the electron energy and the photon frequency and vanishes in the Born limit as well as in the low-frequency limit and for small scattering angles. Outside these regions, $\mathrm{CD}$ has a noticeable magnitude and is quite accessible for experimental observation. It should be noted that $\mathrm{CD}$ in photoprocesses with nonpolarized atomic targets is an essentially quantummechanical interference effect, which is absent, in particular, in the classical analysis of $\mathrm{BrS}$ in a strong laser field [46]. At the same time, numerical quantum calcu- lations of single-photon Coulomb BrS, which are not confined to perturbation theory in the laser field [47] indicate significant $\mathrm{CD}$. Electron scattering by a hydrogen atom in the presence of two fields with linear and circular polarizations is considered in [48]. The emergence of dichroism effects in the presence of the two fields is quite obvious; in this case, CD differs from zero for certain geometries of the fields for fast (Born) electrons also and in the total cross section.

It was noted above that the first Born approximation is insufficient for correctly describing the polarization effect, and the interaction of an electron with the target must be taken into account more exactly; this involves considerable difficulties for processes with two or more photons even in the framework of perturbation theory in the electron interaction with radiation. Since the problem has several vector parameters, the separation of dynamic (depending on the energy and structure of the potential) and kinematic (depending on the photon polarization and the geometry of the problem) factors in the general expressions for cross sections is of prime importance. We will carry out partial-wave analysis of two-photon free-free transitions as applied to potential scattering $U(r)$ both in the field and by atoms with nonzero angular momentum. General results are illustrated by analytic and numerical calculations for scattering by a Coulomb potential. In Section 2, the analytic expression for the amplitude of two-photon dipole transitions is simplified to the maximum possible extent for the case of a central potential $U(r)$, which generalizes results (3) and (4) to the case of single-photon $\mathrm{BrS}$. In contrast to binomial expression (3), the two-photon amplitude in the general case of different photons can be written in the form of five products of invariant (independent of photon polarizations) atomic parameters $Q_{i}$ and the scalar products of photon polarization vectors by the initial and final electron momenta. By analogy with Eq. (4), parameters $Q_{i}$ are presented in the form of a series of products of the second-order radial MEs $M_{l^{\prime} l}^{L=l \pm 1}$ between the states of the continuum with fixed values of the orbital angular momenta $l$ and $l$ and the Legendre polynomials in the scattering angle $\theta$. Such a form of representing the amplitude makes it possible to obtain explicit expressions for the atomic parameters that describe dichroic polarization effects depending on the sign of the photon helicity (Subsection 2.3) and to demonstrate, in particular, a new dichroic effect, viz., elliptic dichroism (ED), which emerges in induced two-photon processes along with $\mathrm{CD}$ and vanishes in the case of a purely circular laser field polarization. The optimal conditions for the observation of $\mathrm{CD}$ and $\mathrm{ED}$ are analyzed. Closed analytic expressions for the Coulomb MEs $M_{l l}^{L}$ are obtained in Section 3 (see also Appendix B) in the form of the sum of the MEs $d_{l_{2} l_{1}}\left(E^{\prime}, E\right)$ for conventional bremsstrahlung and a single integral of the function ${ }_{2} F_{1}$. In Section 4 , elastic two-photon transitions are considered and it is 
shown that singularities appearing in $M_{l^{\prime} l}^{L}$ in the case when the energy difference between the initial and final states becomes insignificant $\left(E^{\prime} \longrightarrow E\right)$ are compensated in the calculation of the invariant parameters $Q_{i}^{e l}$, for which explicit analytic expressions are derived in the case of Coulomb scattering. The limiting ranges of low and high photon frequencies are investigated, and simple asymptotic forms of inelastic scattering amplitudes are obtained for the central field $U(r)$ in Section 5. The frequency and energy dependences of the Coulomb radial MEs and the accuracy of approximate methods of computation are discussed in Subsection 6.1. The results for the angular distributions and the polarization dependence of induced two-photon emission and absorption are given in Subsection 6.2, where the numerical values of the cross sections of oneand two-photon bremsstrahlung transitions are compared. The analytic formulas derived by us for the Coulomb MEs $M_{l^{\prime} l}^{L}$ are the most general expressions for the amplitudes of two-photon transitions in the Coulomb field between the states with fixed orbital angular momenta $l$ and $l$. It is shown in Appendix B that the well-known results for the case when both states or one state belong to the discrete spectrum can be obtained from these formulas by the analytic continuation in energy $\left(p \longrightarrow i m Z e^{2} / n \hbar\right.$ and/or $\left.p^{\prime} \longrightarrow i m Z e^{2} / n^{\prime} \hbar\right)$ [49].

In the subsequent analysis, we will use the atomic system of units.

\section{POLARIZATION-ANGULAR STRUCTURE OF CROSS SECTIONS OF TWO-PHOTON BREMSSTRAHLUNG PROCESSES}

\subsection{General Formulas}

The amplitude of a two-photon electron transition between two scattering states with asymptotic momenta $\mathbf{p}_{i} \equiv \mathbf{p}$ and $\mathbf{p}_{f} \equiv \mathbf{p}^{\prime}$ in potential $U(r)$ is determined by the second-order ME in perturbation theory (cf. Eq. (2)):

$$
\begin{gathered}
M\left(\mathbf{e}_{2}, \mathbf{e}_{1}, \mathscr{E}\right) \\
=-\left\langle\psi_{\mathbf{p}^{\prime}}^{(-)}\left|\left(\mathbf{e}_{2} \cdot \nabla^{\prime}\right) G_{\mathscr{E}}\left(\mathbf{r}^{\prime}, \mathbf{r}\right)\left(\mathbf{e}_{1} \cdot \nabla\right)\right| \psi_{\mathbf{p}}^{(+)}\right\rangle,
\end{gathered}
$$

where $\psi_{\mathbf{p}^{\prime}}^{(-)}(\mathbf{r})$ and $\psi_{\mathbf{p}}^{(+)}(\mathbf{r})$ are the wave functions of the continuum with the asymptotic forms of converging (-) and diverging $(+)$ waves, normalized by the condition

$$
\left\langle\psi_{\mathbf{p}^{\prime}}^{( \pm)} \mid \psi_{\mathbf{p}}^{( \pm)}\right\rangle=(2 \pi)^{3} \delta\left(\mathbf{p}^{\prime}-\mathbf{p}\right),
$$

and $G_{\mathscr{C}}$ is Green's function of the Hamiltonian with potential $U(r)$ and with the asymptotic form for diverging waves for $\mathscr{E}>0$. The differential cross section of a spontaneous $2 \mathrm{BrS}$ with the photon emission in directions $\mathbf{k}_{1}$ and $\mathbf{k}_{2}\left(\left|\mathbf{k}_{i}\right|=1, \mathbf{e}_{i} \cdot \mathbf{e}_{i}^{*}=1, i=1,2\right)$ has the form

$$
\begin{gathered}
\frac{d \sigma^{5}}{d \Omega_{\mathbf{p}^{\prime}} d \Omega_{\mathbf{k}_{1}} d \Omega_{\mathbf{k}_{2}} d \omega_{1} d \omega_{2}}=\frac{\alpha^{6}}{(2 \pi)^{6}} \frac{p^{\prime}}{p} \omega_{1} \omega_{2} \\
\times\left|M\left(\mathbf{e}_{2}^{*}, \mathbf{e}_{1}^{*}, E-\omega_{1}\right)+M\left(\mathbf{e}_{1}^{*}, \mathbf{e}_{2}^{*}, E-\omega_{2}\right)\right|^{2} .
\end{gathered}
$$

For an electron scattered by a force center $U(r)$ in the presence of a high-intensity light wave whose electric vector is written in the form

$$
\mathbf{F}(t)=F \operatorname{Re}\{\operatorname{eexp}(-i \omega t)\}, \quad \mathbf{e} \cdot \mathbf{e}^{*}=1,
$$

induced multiphoton processes are of prime importance. Induced two-photon transitions determine double bremsstrahlung emission and absorption as well as the correction to the elastic scattering cross section, which is linear in the wave intensity $I=c F^{2} / 4 \pi$. The cross sections of the above-mentioned processes are also determined by ME (7). For example, the differential (with respect to the angles of a scattered electron) induced $2 \mathrm{BrS}$ cross section has the form

$$
\frac{d \sigma}{d \Omega_{\mathbf{p}^{\prime}}}=\frac{1}{4 \pi^{2}}\left(\frac{F}{2 \omega}\right)^{4} \frac{p^{\prime}}{p}\left|M\left(\mathbf{e}^{*}, \mathbf{e}^{*}, \mathscr{E}\right)\right|^{2},
$$

where the electron energy $\mathscr{E}$ in the intermediate state is connected to the energies in the initial $\left(E=p^{2} / 2\right)$ and final $\left(E^{\prime}=p^{\prime 2} / 2\right)$ states through the relation $\mathscr{E}=E-\omega=$ $E^{\prime}+\omega$. Similarly, the double bremsstrahlung absorption cross section is given by

$$
\frac{d \sigma}{d \Omega_{\mathbf{p}^{\prime}}}=\frac{1}{4 \pi^{2}}\left(\frac{F}{2 \omega}\right)^{4} \frac{p^{\prime}}{p}|M(\mathbf{e}, \mathbf{e}, \mathscr{E})|^{2},
$$

where $\mathscr{E}=E+\omega=E^{\prime}-\omega$.

In the case of elastic scattering $\left(E^{\prime}=E\right)$, the inclusion of the interaction with the light wave in the lowest (second) order of perturbation theory gives a correction to the amplitude linear in wave intensity,

$$
f=f_{0}+f_{2},
$$

where $f_{0}$ is the amplitude of elastic scattering by potential $U(r)$ in the absence of a light wave, while

$$
f_{2}=\frac{1}{2 \pi}\left(\frac{F}{2 \omega}\right)^{2}
$$

$$
\times\left[M\left(\mathbf{e}, \mathbf{e}^{*}, E-\omega\right)+M\left(\mathbf{e}^{*}, \mathbf{e}, E+\omega\right)\right]
$$

is the correction associated with the two-photon transition induced by the wave (reemission of a photon by an electron during scattering, Fig. 1). A correction on the order of $F^{2}$ to the elastic scattering cross section is determined by the interference of amplitudes $f_{0}$ and $f_{2}$ :

$$
\frac{d \sigma}{d \Omega_{\mathbf{p}^{\prime}}}=\left|f_{0}\right|^{2}+2 \operatorname{Re}\left(f_{0}^{*} f_{2}\right)
$$




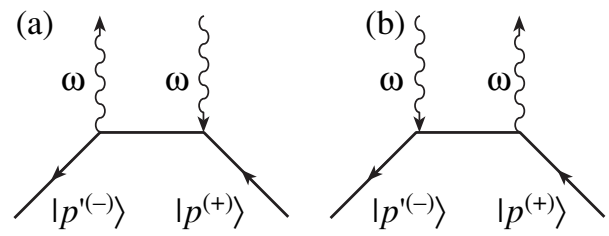

Fig. 1. Feynman diagrams for reemission of a photon by an electron in the continuum.

\subsection{Partial-Wave Expansion of the Amplitude}

Since $\mathbf{e}_{1}$ and $\mathbf{e}_{2}$ appear linearly in Eq. (7), ME $M\left(\mathbf{e}_{2}, \mathbf{e}_{1}, \mathscr{E}\right)$ can be presented as the sum of the products of linearly independent combinations of vectors $\mathbf{e}_{1}, \mathbf{e}_{2}$ and $\mathbf{n}, \mathbf{n}^{\prime}$ (where $\mathbf{n}=\mathbf{p} / p$ and $\mathbf{n}^{\prime}=\mathbf{p}^{\prime} / p^{\prime}$ ) and the invariant amplitudes $\mathscr{P}_{i}$ depending only on $p, p^{\prime}, \mathscr{E}$, and the angle $\theta$ between $\mathbf{n}$ and $\mathbf{n}$ '. There are five linearly independent combinations $\mathbf{e}_{1}, \mathbf{e}_{2}$ and $\mathbf{n}, \mathbf{n}^{\prime}$; choosing these combinations appropriately, we can write $M\left(\mathbf{e}_{2}, \mathbf{e}_{1}, \mathscr{E}\right)$ in the form

$$
\begin{gathered}
M\left(\mathbf{e}_{2}, \mathbf{e}_{1}, \mathscr{E}\right)=\mathscr{P}_{1}\left(\mathbf{e}_{2} \cdot \mathbf{n}\right)\left(\mathbf{e}_{1} \cdot \mathbf{n}\right) \\
+\mathscr{P}_{2}\left(\mathbf{e}_{2} \cdot \mathbf{n}^{\prime}\right)\left(\mathbf{e}_{1} \cdot \mathbf{n}^{\prime}\right)+\mathscr{P}_{3}\left(\mathbf{e}_{2} \cdot \mathbf{n}\right)\left(\mathbf{e}_{1} \cdot \mathbf{n}^{\prime}\right) \\
+\mathscr{P}_{4}\left(\mathbf{e}_{2} \cdot \mathbf{n}^{\prime}\right)\left(\mathbf{e}_{1} \cdot \mathbf{n}\right)+\mathscr{P}_{5}\left(\mathbf{e}_{2} \cdot \mathbf{e}_{1}\right) .
\end{gathered}
$$

It should be noted that the analytic expressions for the amplitude of two-photon transitions in the Coulomb field, derived in [11-13] without using partial expansions, have exactly the same structure. The explicit form of the amplitudes $\mathscr{P}_{i}=\mathscr{P}_{i}\left(p, p^{\prime}, \theta, \mathscr{E}\right)$ for an arbitrary potential $U(r)$ can be obtained only by specific calculations of ME (7). A general method for simplifying expressions of form (7) is the use of multipole expansions of the wave functions and operators appearing in Eq. (7), followed by integration with respect to angular variables by the methods of quantum theory for angular momentum [50]. Let us write the wave functions and Green's function in Eq. (7) in the form of expansions in spherical functions:

$$
\begin{gathered}
\psi_{\mathbf{p}}^{( \pm)}(\mathbf{r})=\frac{(2 \pi)^{3 / 2}}{\sqrt{p}} \\
\times \sum_{l m} i^{l} \exp \left( \pm i \delta_{l}(p)\right) R_{E l}(r) Y_{l m}\left(\frac{\mathbf{r}}{r}\right) Y_{l m}^{*}(\mathbf{n}), \\
G_{\mathscr{E}}\left(\mathbf{r}, \mathbf{r}^{\prime}\right)=\sum_{L M} g_{L}\left(r, r^{\prime}, \mathscr{E}\right) Y_{L M}\left(\frac{\mathbf{r}}{r}\right) Y_{L M}^{*}\left(\frac{\mathbf{r}^{\prime}}{r^{\prime}}\right),
\end{gathered}
$$

where $R_{E l}(r)$ are the radial functions of the continuum in potential $U(r)$, normalized to energy, and $\delta_{l}(p)$ are scattering phases. Substituting expressions (15) into Eq. (7), integrating with respect to angles, and carrying out summation over the angular momenta components, we obtain

$$
\begin{gathered}
M\left(\mathbf{e}_{2}, \mathbf{e}_{1}, \mathscr{E}\right)=-\frac{(2 \pi)^{3}}{\sqrt{p p^{\prime}}} \\
\times \sum_{c=0}^{2} \sum_{l l^{\prime} L} i^{l+l^{\prime}} \exp \left[i\left(\delta_{l^{\prime}}\left(p^{\prime}\right)+\delta_{l}(p)\right)\right] \\
\times\left\{\begin{array}{lll}
1 & 1 & c \\
l^{\prime} & l & L
\end{array}\right\}\left\langle R_{E^{\prime} l^{\prime}}\left\|\nabla g_{L} \nabla\right\| R_{E l}\right\rangle \\
\times\left(\left\{\mathbf{e}_{2} \otimes \mathbf{e}_{1}\right\}_{c} \cdot\left\{Y_{l^{\prime}}\left(\mathbf{n}^{\prime}\right) \otimes Y_{l}(\mathbf{n})\right\}_{c}\right)
\end{gathered}
$$

(we will use below the standard notation in the quantum theory of angular momentum [50]).

Expressions of type (16) are usually considered as the final result of analytic transformations of the "geometrical" part of the amplitudes by the methods of quantum theory of angular momentum, and subsequent calculations are based on numerical computations of tensor constructions in Eq. (16) in an appropriately chosen system of coordinates (see, for example, the publications [30] on the induced one- and two-photon emission and absorption in a linearly polarized field; it should be noted that the function $\psi_{\mathbf{p}^{\prime}}^{( \pm)}(\mathbf{r})$ with the asymptotic form for diverging waves is groundlessly used by the author of [30] as the final state of ME (2) and (7)). A special technique for simplifying tensor products of the spherical functions $Y_{l m}(\mathbf{n})$ based on the reduced formula for bipolar harmonics $Y_{L M}^{l^{\prime} l}\left(\mathbf{n}^{\prime}, \mathbf{n}\right)$ defined as

$$
\begin{gathered}
Y_{L M}^{l^{\prime \prime} l}\left(\mathbf{n}^{\prime}, \mathbf{n}\right)=\left\{Y_{l^{\prime}}\left(\mathbf{n}^{\prime}\right) \otimes Y_{l}(\mathbf{n})\right\}_{L M} \\
=\sum_{m m^{\prime}} C_{l^{\prime} m^{\prime} l m}^{L M} Y_{l^{\prime} m^{\prime}}\left(\mathbf{n}^{\prime}\right) Y_{l m}(\mathbf{n})
\end{gathered}
$$

was developed in [45] (see also [51]). This technique makes it possible to present $Y_{L M}^{k, L-k}\left(\mathbf{n}^{\prime}, \mathbf{n}\right)$ with arbitrary values of $l, l$ ' $>L$ in the form of a finite sum of "minimal" harmonics $Y_{L M}^{k, L-k}\left(\mathbf{n}^{\prime}, \mathbf{n}\right)$ with $0 \leq k \leq L$ and Legendre polynomials. For example, for the bipolar harmonic $Y_{2 m}^{l l}\left(\mathbf{n}^{\prime}, \mathbf{n}\right)$, we have [45]

$$
\begin{aligned}
Y_{2 m}^{l l}\left(\mathbf{n}^{\prime}, \mathbf{n}\right)= & \frac{(-1)^{l-1}}{4 \pi}\left[\frac{30(2 l+1)}{(2 l-1) l(l+1)(2 l+3)}\right]^{1 / 2} \\
& \times\left(P_{l}^{(1)}(x)\left\{\mathbf{n}^{\prime} \otimes \mathbf{n}\right\}_{2 m}\right. \\
+ & \left.P_{l}^{(2)}(x)\left\{\left[\mathbf{n}^{\prime} \times \mathbf{n}\right] \otimes\left[\mathbf{n}^{\prime} \times \mathbf{n}\right]\right\}_{2 m}\right),
\end{aligned}
$$

where $x=\mathbf{n} \cdot \mathbf{n}^{\prime}=\cos \theta$ and $P_{l}^{(k)}(x)=(d / d x)^{k} P_{l}(x)$. The expressions for the remaining bipolar harmonics $Y_{c m}^{l^{\prime \prime l}}\left(\mathbf{n}^{\prime}, \mathbf{n}\right)$ with $c=0,1,2$, which appear in Eq. (16), are also given in [45]. Using these expressions and writing the appearing tensor constructions in the form $\left(\left\{\mathbf{e}_{2} \otimes\right.\right.$ 
$\left.\left.\mathbf{e}_{1}\right\}_{c} \cdot\left\{\mathbf{n}^{\prime} \otimes \mathbf{n}\right\}_{c}\right)$ in terms of the scalar products of vectors $\mathbf{e}_{1}, \mathbf{e}_{2}$, and $\mathbf{n}$, n' (Section 3 in [50]), we can reduce expression (16) for $M\left(\mathbf{e}_{2}, \mathbf{e}_{1}, \mathscr{E}\right)$ to the form (14). It is convenient to write the final result in the form

$$
\begin{gathered}
M\left(\mathbf{e}_{2}, \mathbf{e}_{1}, \mathscr{E}\right)=Q_{1}\left[\left(\mathbf{e}_{2} \cdot \mathbf{n}\right)\left(\mathbf{e}_{1} \cdot \mathbf{n}\right)-\frac{1}{3}\left(\mathbf{e}_{2} \cdot \mathbf{e}_{1}\right)\right] \\
+Q_{2}\left[\left(\mathbf{e}_{2} \cdot \mathbf{n}^{\prime}\right)\left(\mathbf{e}_{1} \cdot \mathbf{n}^{\prime}\right)-\frac{1}{3}\left(\mathbf{e}_{2} \cdot \mathbf{e}_{1}\right)\right] \\
+\frac{1}{2} Q_{3}\left[\left(\mathbf{e}_{2} \cdot \mathbf{n}^{\prime}\right)\left(\mathbf{e}_{1} \cdot \mathbf{n}\right)+\left(\mathbf{e}_{2} \cdot \mathbf{n}\right)\left(\mathbf{e}_{1} \cdot \mathbf{n}^{\prime}\right)\right. \\
\left.\quad-\frac{2}{3}\left(\mathbf{n} \cdot \mathbf{n}^{\prime}\right)\left(\mathbf{e}_{2} \cdot \mathbf{e}_{1}\right)\right] \\
+Q_{4}\left[\left(\mathbf{e}_{2} \cdot \mathbf{n}^{\prime}\right)\left(\mathbf{e}_{1} \cdot \mathbf{n}\right)-\left(\mathbf{e}_{2} \cdot \mathbf{n}\right)\left(\mathbf{e}_{1} \cdot \mathbf{n}^{\prime}\right)\right]+Q_{5}\left(\mathbf{e}_{2} \cdot \mathbf{e}_{1}\right)
\end{gathered}
$$

where

$$
\begin{gathered}
Q_{1}=-\sum_{l=2}^{\infty}\left[\frac{1}{2 l+3}\left(M_{l l}^{l+1}+M_{l+2 l}^{l+1}\right)\right. \\
\left.+\frac{1}{2 l-1}\left(M_{l l}^{l-1}+M_{l-2 l}^{l-1}\right)\right] P_{l}^{(2)}(x), \\
Q_{2}=-\sum_{l=2}^{\infty}\left[\frac{1}{2 l+3}\left(M_{l l}^{l+1}+M_{l l+2}^{l+1}\right)\right. \\
\left.+\frac{1}{2 l-1}\left(M_{l l}^{l-1}+M_{l l-2}^{l-1}\right)\right] P_{l}^{(2)}(x), \\
Q_{3}=\sum_{l=1}^{\infty}\left[\frac{1}{2 l-1} M_{l l}^{l-1}+\frac{1}{2 l+3} M_{l l}^{l+1}\right] P_{l}^{(1)}(x) \\
+2 \sum_{l=2}^{\infty}\left[\left(\frac{1}{2 l-1} M_{l l}^{l-1}+\frac{1}{2 l+3} M_{l l}^{l+1}\right) x\right. \\
\left.+\frac{1}{2 l+1}\left(M_{l-1}^{l}{ }^{l+1}+M_{l+1 l-1}^{l}\right)\right] P_{l}^{(2)}(x), \\
Q_{5}=\frac{1}{3} \sum_{l=0}^{\infty}\left[l M_{l l}^{l-1}+(l+1) M_{l l}^{l+1}\right] P_{l}(x) . \\
Q_{4}=\frac{1}{2} \sum_{l=1}^{\infty}\left[M_{l l}^{l-1}-M_{l l}^{l+1}\right] P_{l}^{(1)}(x),
\end{gathered}
$$

Radial MEs $M_{l^{\prime} l}^{L}$ contain the phase factors from relations (15):

$$
M_{l^{\prime} l}^{L}=\left(2 \pi^{2} / \sqrt{p p^{\prime}}\right)
$$

$$
\begin{gathered}
\times \exp \left[i\left(\delta_{l^{\prime}}\left(p^{\prime}\right)+\delta_{l}(p)\right)\right] M_{l^{\prime} l}^{L}\left(E^{\prime}, E, \mathscr{E}\right), \\
M_{l^{\prime} l}^{L}\left(E^{\prime}, E, \mathscr{E}\right) \\
=\left\langle D\left(L, l^{\prime}\right) R_{E l^{\prime}}\left|g_{L}(\mathscr{E})\right| D(L, l) R_{E l}\right\rangle, \\
D\left(l_{1}, l_{2}\right)=\frac{d}{d r}+\frac{\operatorname{sgn}\left(l_{2}-l_{1}\right) \max \left(l_{1}, l_{2}\right)+1}{r} .
\end{gathered}
$$

Formulas (20) give five invariant parameters $Q_{i}$ (which are obviously associated with $\mathscr{P}_{i}$ in Eq. (14)) in explicit form in terms of radial MEs. Relations (20) directly demonstrate the symmetry properties,

$Q_{1}\left(p, p^{\prime}\right)=Q_{2}\left(p^{\prime}, p\right), \quad Q_{3,4,5}\left(p^{\prime}, p\right)=Q_{3,4,5}\left(p, p^{\prime}\right)$.

\subsection{Effects of Circular and Elliptic Dichroism in free-free Transitions}

Parametrization of the amplitude in the form (19) and (20) makes it possible to completely analyze the polarization and angular dependences both in spontaneous $2 \mathrm{BrS}$ (with different polarizations $\mathbf{e}_{1}$ and $\mathbf{e}_{2}$ ) and for induced processes (with $\mathbf{e}_{1}=\mathbf{e}_{2}$ ). Let us first consider induced $2 \mathrm{BrS}$ (the results for double bremsstrahlung absorption follow from the formulas given below with the substitution $\left.\mathbf{e}, \omega \longrightarrow \mathbf{e}^{*},-\omega\right)$. Assuming that $\mathbf{e}_{1}=$ $\mathbf{e}_{2}=\mathbf{e}^{*}$ in Eq. (19), we see that parameter $Q_{4}$ for induced processes is omitted and the expression for $M$ has the form

$$
\begin{gathered}
M=Q_{1}\left(\mathbf{e}^{*} \cdot \mathbf{n}\right)^{2}+Q_{2}\left(\mathbf{e}^{*} \cdot \mathbf{n}^{\prime}\right)^{2} \\
+Q_{3}\left(\mathbf{e}^{*} \cdot \mathbf{n}\right)\left(\mathbf{e}^{*} \cdot \mathbf{n}^{\prime}\right)+2\left(\mathbf{e}^{*} \cdot \mathbf{e}^{*}\right), \\
2=Q_{5}-\frac{1}{3}\left(Q_{1}+Q_{2}+x Q_{3}\right) .
\end{gathered}
$$

As a result, cross section (9) assumes the form

$$
\frac{d \sigma}{d \Omega_{\mathbf{p}^{\prime}}}=\frac{1}{4 \pi^{2}}\left(\frac{F}{2 \omega}\right)^{4} \frac{p^{\prime}}{p}\left(f_{\mathrm{reg}}+\Delta_{\mathrm{CD}}+\Delta_{\mathrm{ED}}\right),
$$

where

$$
\begin{aligned}
f_{\text {reg }}= & \left|Q_{1}\right|^{2}|\mathbf{e} \cdot \mathbf{n}|^{4}+\left|Q_{2}\right|^{2}\left|\mathbf{e} \cdot \mathbf{n}^{\prime}\right|^{4}+\left|Q_{3}\right|^{2}|\mathbf{e} \cdot \mathbf{n}|^{2}\left|\mathbf{e} \cdot \mathbf{n}^{\prime}\right|^{2} \\
+ & l^{2}|2|^{2}+2 \operatorname{Re}\left(Q_{1}^{*} Q_{2}\right) \operatorname{Re}\left\{(\mathbf{e} \cdot \mathbf{n})^{2}\left(\mathbf{e}^{*} \cdot \mathbf{n}^{\prime}\right)^{2}\right\} \\
& +2 \operatorname{Re}\left(Q_{1}^{*} Q_{3}\right)|\mathbf{e} \cdot \mathbf{n}|^{2} \operatorname{Re}\left\{(\mathbf{e} \cdot \mathbf{n})\left(\mathbf{e}^{*} \cdot \mathbf{n}^{\prime}\right)\right\} \\
& +2 l \operatorname{Re}\left(Q_{1}^{*} 2\right) \operatorname{Re}\left\{(\mathbf{e} \cdot \mathbf{n})^{2}\right\} \\
& +2 \operatorname{Re}\left(Q_{2}^{*} Q_{3}\right)\left|\mathbf{e} \cdot \mathbf{n}^{\prime}\right|^{2} \operatorname{Re}\left\{\left(\mathbf{e} \cdot \mathbf{n}^{\prime}\right)\left(\mathbf{e}^{*} \cdot \mathbf{n}\right)\right\} \\
& +2 l \operatorname{Re}\left(Q_{2}^{*} 2\right) \operatorname{Re}\left\{\left(\mathbf{e} \cdot \mathbf{n}^{\prime}\right)^{2}\right\} \\
& +2 l \operatorname{Re}\left(Q_{3}^{*} 2\right) \operatorname{Re}\left\{(\mathbf{e} \cdot \mathbf{n})\left(\mathbf{e} \cdot \mathbf{n}^{\prime}\right)\right\},
\end{aligned}
$$




$$
\begin{aligned}
& \Delta_{\mathrm{CD}}= 2 \operatorname{Im}\left\{(\mathbf{e} \cdot \mathbf{n})\left(\mathbf{e}^{*} \cdot \mathbf{n}^{\prime}\right)\right\}\left(\operatorname{Im}\left(Q_{2}^{*} Q_{3}\right)\left|\mathbf{e} \cdot \mathbf{n}^{\prime}\right|^{2}\right. \\
&\left.-2 \operatorname{Im}\left(Q_{1}^{*} Q_{2}\right) \operatorname{Re}\left\{(\mathbf{e} \cdot \mathbf{n})\left(\mathbf{e}^{*} \cdot \mathbf{n}^{\prime}\right)\right\}-\operatorname{Im}\left(Q_{1}^{*} Q_{3}\right)|\mathbf{e} \cdot \mathbf{n}|^{2}\right) \\
& \Delta_{\mathrm{ED}}=-2 l\left(\operatorname{Im}\left(Q_{1}^{*} 2\right) \operatorname{Im}\left\{(\mathbf{e} \cdot \mathbf{n})^{2}\right\}\right. \\
& \quad+\operatorname{Im}\left(Q_{2}^{*} 2\right) \operatorname{Im}\left\{\left(\mathbf{e} \cdot \mathbf{n}^{\prime}\right)^{2}\right\} \\
&\left.+\operatorname{Im}\left(Q_{3}^{*} 2\right) \operatorname{Im}\left\{(\mathbf{e} \cdot \mathbf{n})\left(\mathbf{e} \cdot \mathbf{n}^{\prime}\right)\right\}\right)
\end{aligned}
$$

Obviously, $f_{\text {reg }}$ remains unchanged upon the substitution $\mathbf{e} \rightleftarrows \mathbf{e}^{*}$, i.e., is independent of the sign of photon helicity. In order to analyze $\Delta_{\mathrm{CD}}$ and $\Delta_{\mathrm{ED}}$, it is convenient to write the unit complex polarization vector in an invariant (relative to the choice of the coordinate system) form,

$$
\mathbf{e}=\frac{\boldsymbol{\epsilon}+i \eta[\mathbf{k} \times \boldsymbol{\epsilon}]}{\sqrt{1+\eta^{2}}}, \quad-1 \leq \eta \leq 1,
$$

where the unit vectors $\boldsymbol{\epsilon}$ and $\mathbf{k}$ define the directions of the principal axis of the polarization ellipse and of the propagation of waves, while ellipticity $\eta$ is connected to the degrees of linear $(l)$ and circular $(\xi)$ polarizations, which we define as in [3]:

$$
\begin{aligned}
& l=\frac{1-\eta^{2}}{1+\eta^{2}}=\mathbf{e} \cdot \mathbf{e}=\mathbf{e}^{*} \cdot \mathbf{e}^{*}, \\
& \xi=\frac{2 \eta}{1+\eta^{2}}=i \mathbf{k} \cdot\left[\mathbf{e}^{*} \times \mathbf{e}\right] .
\end{aligned}
$$

Using relations (28), we can easily find that

$$
\begin{gathered}
2 \operatorname{Im}\left\{\left(\mathbf{e}^{*} \cdot \mathbf{n}\right)\left(\mathbf{e} \cdot \mathbf{n}^{\prime}\right)\right\}=\xi\left(\mathbf{k} \cdot\left[\mathbf{n} \times \mathbf{n}^{\prime}\right]\right), \\
2 \operatorname{Im}\left\{(\mathbf{e} \cdot \mathbf{n})\left(\mathbf{e} \cdot \mathbf{n}^{\prime}\right)\right\}=\xi\left\{(\boldsymbol{\epsilon} \cdot \mathbf{n})\left([\mathbf{k} \times \mathbf{\epsilon}] \cdot \mathbf{n}^{\prime}\right)\right. \\
\left.+\left(\boldsymbol{\epsilon} \cdot \mathbf{n}^{\prime}\right)([\mathbf{k} \times \boldsymbol{\epsilon}] \cdot \mathbf{n})\right\},
\end{gathered}
$$

so that

$$
\Delta_{\mathrm{CD}} \sim \xi, \quad \Delta_{\mathrm{ED}} \sim \xi l .
$$

Relations (30) show that the last two terms in Eq. (24) lead to a dependence of the cross section on the sign of $\xi$ and describe the CD and ED effects. The value of $\Delta_{\mathrm{ED}}$ vanishes for purely circular polarization $(\xi= \pm 1, l=0)$, while the $\mathrm{CD}$ term $\Delta_{\mathrm{CD}}$ attains its maximum value in this case. Although the quantitative results for $\mathrm{CD}$ and $\mathrm{ED}$ can be obtained only from numerical calculations, the distinguishing features of these effects can be seen even from general formulas (24)-(27). In particular, the CD and ED terms in Eq. (24) exhibit essentially different dependences not only on the polarization parameters of the waves, but also on the geometry of the process. For example, the term $\Delta_{\mathrm{CD}}$ contains the common polarization-angular factor $\xi\left(\mathbf{k} \cdot\left[\mathbf{n} \times \mathbf{n}^{\prime}\right]\right)$ (see relations (29)) and attains the "geometrical" maximum for a light wave propagat- ing orthogonally to the plane of electron scattering: $\mathbf{k} \| \pm[\mathbf{n n}]$. In this case, the term $\Delta_{C D}$ has the form

$$
\begin{gathered}
\Delta_{\mathrm{CD}}=\xi \sin \theta \\
\times\left[2 \operatorname{Im}\left(Q_{1}^{*} Q_{2}\right) \cos \theta+\operatorname{Im}\left(Q_{1}^{*} Q_{3}\right)-\operatorname{Im}\left(Q_{2}^{*} Q_{3}\right)\right] .
\end{gathered}
$$

If, however, the initial electron momentum is collinear to the direction of wave propagation $(\mathbf{n} \times \mathbf{k}=0)$, CD vanishes in the same way as in the case of forward and backward scattering, $\mathbf{n}^{\prime}= \pm \mathbf{n}$. It should be noted that the kinematic factor $\xi(\mathbf{k} \cdot[\mathbf{a} \times \mathbf{b}])$ determining $\Delta_{\mathrm{CD}}$ is universal by nature and describes $C D$ in various singlephonon processes with nonpolarized atoms in the presence of two polar vectors, $\mathbf{a}$ and $\mathbf{b}$, say, in the case of conventional and induced single-photon $\mathrm{BrS}$ [4] and two-electron ionization of an atom by a hard photon $[45,52]$. ED is possible only in processes with two or more identical photons and is less sensitive to the process geometry; in accordance with Eq. (27), the necessary condition for vanishing $\Delta_{\mathrm{CD}}$ is simultaneous fulfillment of the conditions $\mathbf{e} \cdot \mathbf{n}=0$ and $\mathbf{e} \cdot \mathbf{n}^{\prime}=0$. The ED effect is manifested in "pure form" (without CD accompanying it) in the angular distribution of scattered electrons, when the initial momentum $\mathbf{p}$ is collinear to the light beam, $[\mathbf{k} \times \mathbf{n}]=0$. In this case, only the terms with 2 and $Q_{2}$ remain in relation (23) and $\Delta_{\mathrm{ED}}$ has the simple form

$$
\begin{aligned}
& \Delta_{\mathrm{ED}}=-2 l \operatorname{Im}\left(Q_{2}^{*} \mathscr{2}\right) \operatorname{Im}\left\{\left(\mathbf{e} \cdot \mathbf{n}^{\prime}\right)^{2}\right\} \\
= & -2 l \xi \operatorname{Im}\left(Q_{2}^{*} \mathscr{2}\right)\left(\boldsymbol{\epsilon} \cdot \mathbf{n}^{\prime}\right)\left(\mathbf{k} \cdot\left[\boldsymbol{\epsilon} \times \mathbf{n}^{\prime}\right]\right)
\end{aligned}
$$

and attains its maximum value for scattering at right angles (i.e., in the polarization plane) in the directions forming the angles $\pm \pi / 4$ and $\pm 3 \pi / 4$ with the direction of the principal axis of the polarization ellipse.

In contrast to $\mathrm{CD}$, the ED effect persists in the cross section of the induced 2BrS (or double bremsstrahlung absorption), integrated over the directions n' of a scattered electron momentum. In this case, the cross section depends only on vectors $\mathbf{e}$ and $\mathbf{n}$ and has the following structure:

$$
\begin{aligned}
\sigma=\frac{1}{4 \pi^{2}}( & \left.\frac{F}{2 \omega}\right)^{4} \frac{p^{\prime}}{p}\left(A_{1}+A_{2} l^{2}+A_{3} l \operatorname{Re}\left\{(\mathbf{e} \cdot \mathbf{n})^{2}\right\}\right. \\
& +A_{4} l \xi(\boldsymbol{\epsilon} \cdot \mathbf{n})(\boldsymbol{\epsilon} \cdot[\mathbf{n} \times \mathbf{k}]) \\
& \left.+A_{5}|\mathbf{e} \cdot \mathbf{n}|^{2}+A_{6}|\mathbf{e} \cdot \mathbf{n}|^{4}\right) .
\end{aligned}
$$

It can be seen that the ED term in the expression for $\sigma$ attains its maximum value when the initial electron momentum $\mathbf{p}$ is orthogonal to the direction of the light beam and forms an angle of $\pi / 4$ with principal axis of the polarization ellipse. It should be noted that expression (33) is completely identical kinematically to the angular distribution of photoelectrons in the case of two-photon ionization of an atom with a nonzero orbital angular momentum in an elliptically polarized 
field (polarization effects and ED in this problem were analyzed in [53]). We can derive explicit expressions for dynamic parameters $A_{i}$ in relation (33) in terms of radial MEs (21) by integrating the squared modulus of amplitude (16) with respect to $\mathbf{n}^{\prime}$. By way of an example, we consider only the expression for the "dichroic parameter" $A_{4}$ :

$$
\begin{aligned}
A_{4}= & \frac{16 \pi^{5}}{p p^{\prime}} \sum_{l=0}^{\infty} \operatorname{Im}\left\{\frac{l(l+1)}{(2 l-1)(2 l+3)} M_{l l}^{l-1} M_{l l}^{l+1 *}\right. \\
& +\left(l M_{l l}^{l-1}+(l+1) M_{l l}^{l+1}\right) \\
& \times\left[\frac{(l+1)(l+2)}{(2 l+1)(2 l+3)} e^{i\left(\delta_{l}(p)-\delta_{l+2}(p)\right)} M_{l l+2}^{l+1 *}\right. \\
+ & \left.\left.\frac{l(l-1)}{(2 l-1)(2 l+1)} e^{i\left(\delta_{l}(p)-\delta_{l-2}(p)\right)} M_{l l-2}^{l-1 *}\right]\right\} .
\end{aligned}
$$

In all investigated cases, the numerical values of dichroic parameters $\left(\Delta_{C D}, \Delta_{E D}\right.$, and $\left.A_{4}\right)$ in the cross sections is determined in the long run by the relation between the real and imaginary parts of the radial ME $M_{l^{\prime} l}^{L}$, i.e., by the interference of Hermite and anti-Hermite parts of the process amplitude. Consequently, dichroism effects disappear in the Born electron energy range $E$ and $E^{\prime}$ as well as in the low-frequency limit (see Section 5 below, where it is proved that the parameters $Q_{i}$ differ in this limit differ only in the real multipliers so that $\left.\operatorname{Im}\left(Q_{i}^{*} Q_{j}\right)=0\right)$. In the remaining cases, the dichroic terms in the cross section have no smallness parameter symbols and the relative magnitude of the dichroism effects (in a favorable geometry of the process) may be as high as $100 \%$. Thus, in contrast to single-photon bremsstrahlung processes in which only the CD effect can take place and the cross section of elliptical polarization can be reconstructed from experimental data for linear and circular polarizations, the fullest information on the process in the case of double-photon bremsstrahlung emission and absorption can be obtained only from a light wave with an elliptical polarization.

It should be borne in mind in an analysis of polarization effects in elastic scattering that $Q_{1}=Q_{2}$ for $E^{\prime}=E$, and, hence, the correction to the elastic cross section in Eq. (13) contains four invariant parameters $Q_{i}^{\mathrm{el}}$ :

$$
\begin{gathered}
\frac{d \sigma}{d \Omega_{\mathbf{p}^{\prime}}}=\left|f_{0}\right|^{2}+\frac{1}{\pi}\left(\frac{F}{2 \omega}\right)^{2} \\
\times\left\{\operatorname{Re}\left(f_{0}^{*} Q_{1}^{\mathrm{el}}\right)\left(|\mathbf{e} \cdot \mathbf{n}|^{2}+\left|\mathbf{e} \cdot \mathbf{n}^{\prime}\right|^{2}-2 / 3\right)\right. \\
+\operatorname{Re}\left(f_{0}^{*} Q_{2}^{\mathrm{el}}\right)\left(\operatorname{Re}\left\{\left(\mathbf{e}^{*} \cdot \mathbf{n}\right)\left(\mathbf{e} \cdot \mathbf{n}^{\prime}\right)\right\}-(1 / 3) \cos \theta\right) \\
\left.+\operatorname{Re}\left(f_{0}^{*} Q_{4}^{\mathrm{el}}\right)+\operatorname{Im}\left(f_{0}^{*} Q_{3}^{\mathrm{el}}\right) \xi\left(\mathbf{k} \cdot\left[\mathbf{n} \times \mathbf{n}^{\prime}\right]\right)\right\} .
\end{gathered}
$$

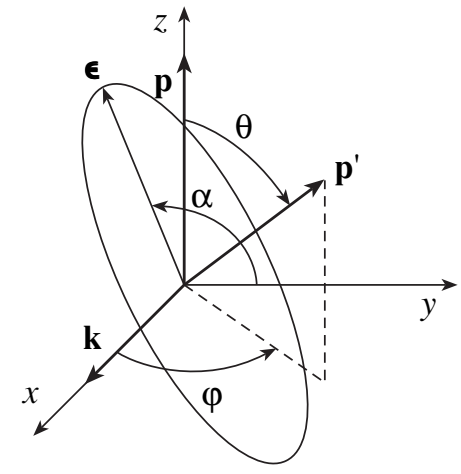

Fig. 2. Geometry of induced bremsstrahlung emission and absorption: $\theta$ and $\varphi$ are the spherical angles of the momentum vector ' $\mathbf{p}^{\prime}$ ' of a scattered electron in the coordinate system with the polar axis along vector $\mathbf{p}$ and the $x$ axis along the direction $\mathbf{k}$ of the laser beam; angle $\alpha$ defines the orientation of the polarization ellipse in the $y z$ plane.

Thus, elastic scattering of an electron in a light field gives rise only to the $\mathrm{CD}$ effect, which is described by the term containing $Q_{3}^{\mathrm{el}}$ and characterized by the same interference nature and the kinematic dependence as $\mathrm{CD}$ in inelastic processes as well as in the case of single-photon scattering [4]. Explicit expressions for the parameters $Q_{i}^{\text {el }}$ similar to expressions (20) will be given below (see Eqs. (48)).

In view of the presence of several vector parameters in the problem, the symmetry properties of angular distributions of scattered electrons in the general case of elliptic photon polarization can be established only for the simplest initial configurations of the electron and laser beams. The most informative and convenient experimental configuration is the "orthogonal" geometry, in which the initial electron momentum $\mathbf{p}$ ( $z$ axis) is orthogonal to the direction of the light beam ( $x$ axis), while the principal axis of the polarization ellipse forms angle $\alpha$ with the $y$ axis (Fig. 2). In this case, the $y z$ (polarization) plane is the symmetry plane of the angular distribution in the general case of elliptic polarization. Since in the case of circular polarization the results are independent of angle $\alpha$, the angular distribution in the absence of CD would also possess a symmetry relative to the $x z$ plane, i.e., to the substitution $\varphi \longrightarrow$ $-\varphi$. The CD terms break this symmetry since $\xi(\mathbf{k} \cdot[\mathbf{n} \times$ $\left.\left.\mathbf{n}^{\prime}\right]\right)=-\xi \sin \theta \sin \varphi$. Nevertheless, this relation shows that the angular distributions in a circular field are transformed into one another for $\xi=1$ and $\xi=-1$ upon reflection by the $x z$ plane (or rotation through $180^{\circ}$ about the $z$ axis); i.e., the cross section $d \sigma / d \Omega$ is invariant relative to the substitutions $\xi \longrightarrow-\xi$ and $\varphi \longrightarrow-\varphi$. In an elliptic field, symmetry is lowered and the above invariance is preserved only for values of $\alpha$ multiple to $\pi / 2$.

For spontaneous $2 \mathrm{BrS}$, the cross section integrated over the directions n' of a scattered electron is of experimental interest. The general form of the polarization- 
angular dependence of cross sections for this case follows from Eqs. (8) and (19) (cf. Eq. (33)):

$$
\begin{aligned}
& \frac{d \sigma^{4}}{d \Omega_{\mathbf{k}_{1}} d \Omega_{\mathbf{k}_{2}} d \omega_{1} d \omega_{2}}=a_{1}+a_{2}\left|\mathbf{e}_{1} \cdot \mathbf{e}_{2}\right|^{2}+a_{3}\left|\mathbf{e}_{1} \cdot \mathbf{e}_{2}^{*}\right|^{2} \\
& +a_{4}\left|\mathbf{e}_{1} \cdot \mathbf{n}\right|^{2}+a_{5}\left|\mathbf{e}_{2} \cdot \mathbf{n}\right|^{2}+a_{6}\left|\mathbf{e}_{1} \cdot \mathbf{n}\right|^{2}\left|\mathbf{e}_{2} \cdot \mathbf{n}\right|^{2} \\
& \quad+a_{7} \operatorname{Re} I_{1}+a_{8} \operatorname{Re} I_{2}+a_{9} \operatorname{Im} I_{1}+a_{10} \operatorname{Im} I_{2},
\end{aligned}
$$

where

$$
\begin{aligned}
& I_{1}=\left(\mathbf{e}_{1} \cdot \mathbf{n}\right)\left(\mathbf{e}_{2} \cdot \mathbf{n}\right)\left(\mathbf{e}_{1}^{*} \cdot \mathbf{e}_{2}^{*}\right), \\
& I_{2}=\left(\mathbf{e}_{1} \cdot \mathbf{n}\right)\left(\mathbf{e}_{2}^{*} \cdot \mathbf{n}\right)\left(\mathbf{e}_{1}^{*} \cdot \mathbf{e}_{2}\right) .
\end{aligned}
$$

Omitting cumbersome expressions for coefficients $a_{i}$ in terms of radial ME, we note that factors $\operatorname{Im} I_{1}$ and $\operatorname{Im} I_{2}$ in the last two terms in Eq. (35) change their sign upon the simultaneous replacement of the polarization vectors by their complex conjugate, $\mathbf{e}_{1} \rightleftarrows \mathbf{e}_{1}^{*}$ and $\mathbf{e}_{2} \rightleftarrows$ $\mathbf{e}_{2}^{*}$; for this reason, the cross section contains terms linear in $\xi_{1}$ and $\xi_{2}$ and depends on the sign of the degree of circular polarization of photons. Thus, the CD effect in spontaneous $2 \mathrm{BrS}$ is also preserved in the integration over n'. The complex quantities $I_{1}$ and $I_{2}$ can be expressed in terms of the real vectors $\boldsymbol{\epsilon}_{i}$ and $\mathbf{k}_{i}$ for any polarizations $\mathbf{e}_{1}$ and $\mathbf{e}_{2}$ (such expressions can be found in [54]); however, the case when one of the photons is polarized linearly (say, $\mathbf{e}_{2}=\mathbf{e}_{2}^{*} \equiv \boldsymbol{\epsilon}_{2}$ ) is most interesting for CD observation. In this case, $I_{1}=I_{2} \equiv I$, and the kinematic dependence of the CD term in Eq. (35) is given by

$$
2 \operatorname{Im} I=\xi_{1}\left(\boldsymbol{\epsilon}_{2} \cdot \mathbf{n}\right)\left(\boldsymbol{\epsilon}_{2} \cdot\left[\mathbf{n} \times \mathbf{k}_{1}\right]\right) .
$$

This expression has a maximum in the orthogonal geometry ( $\left.\mathbf{k}_{1} \perp \mathbf{n}, \mathbf{k}_{2}=-\mathbf{k}_{1}\right)$ used in experiments [8], when bremsstrahlung photons are detected in the opposite direction at right angles to the incident electron beam. Using polarization-sensitive detectors, the $C D$ effect can be observed in these experiments by measuring the difference in the yields of photons with right and left circular polarizations for a fixed linear polarization of the second photon at an angle of $\pi / 4$ to the plane of vectors $\mathbf{n}, \mathbf{k}_{1}$, and $\mathbf{k}_{2}$.

When an electron is scattered by a freely oriented atom with a nonzero total angular momentum, the polarization structure of the cross section of two-photon bremsstrahlung processes is much more complicated. Indeed, in this case only the cross section (and not the amplitude) of the process is a scalar that can be presented as a combination of scalar and mixed products of photon polarization vectors and electron momenta $\mathbf{p}$ and $\mathbf{p}^{\prime}$. Accordingly, the number of terms in the expression for the cross section, which are determined by linearly independent polarization-angular factors, increases considerably. Nevertheless, the general expression for the cross section in vector form, as well as explicit expressions for dichroic terms represented in terms of reduced MEs of the momentum operator, can be obtained in analogy to the case of potential scattering.

\section{COULOMB TWO-PHOTON RADIAL MATRIX ELEMENTS}

Formulas (20) express parameters $Q_{i}$ in terms of the radial MEs $M_{l^{\prime} l}^{L}\left(E^{\prime}, E, \mathscr{E}\right)$ (21) of transitions between the states of the continuum with fixed values of the orbital angular momentum in potential $U(r)$. In accordance with the dipole selection rules, MEs of the following four types appear in expressions (20):

$$
\begin{gathered}
M_{l l}^{l+1}\left(E^{\prime}, E, \mathscr{E}\right) \\
=\left\langle D(l+1, l) R_{E^{\prime} l}\left|g_{l+1}(\mathscr{E})\right| D(l+1, l) R_{E l}\right\rangle, \\
M_{l+2 l+2}^{l+1}\left(E^{\prime}, E, \mathscr{E}\right) \\
=\left\langle D(l+1, l+2) R_{E^{\prime} l+2}\left|g_{l+1}(\mathscr{E})\right| D(l+1, l+2) R_{E l+2}\right\rangle, \\
M_{l+2 l}^{l+1}\left(E^{\prime}, E, \mathscr{E}\right) \\
=\left\langle D(l+1, l+2) R_{E^{\prime} l+2}\left|g_{l+1}(\mathscr{E})\right| D(l+1, l) R_{E l}\right\rangle, \\
M_{l l+2}^{l+1}\left(E^{\prime}, E, \mathscr{E}\right) \\
=\left\langle D(l+1, l) R_{E^{\prime} l}\left|g_{l+1}(\mathscr{E})\right| D(l+1, l+2) R_{E l+2}\right\rangle .
\end{gathered}
$$

It can be seen from the definition that $M_{l l+2}^{l+1}(E, E, \mathscr{E})=$ $M_{l+2 l}^{l+1}\left(E, E^{\prime}, \mathscr{E}\right)$; consequently, it is sufficient to calculate $M_{l l}^{l+1}, M_{l+2 l+2}^{l+1}$, and $M_{l+2 l}^{l+1}$ only.

In the case of the Coulomb potential $U(r)=-Z / r$, the MEs $M_{l^{\prime} l}^{L}$ can be calculated in closed analytic form. It is shown in Appendix A that each ME from $M_{l^{\prime} l}^{L}$ can be presented as a sum of six terms of which two contain first-order dipole MEs,

$$
d_{l^{\prime} l}\left(E^{\prime}, E\right)=\left\langle R_{E^{\prime} l^{\prime}}\left|D\left(l^{\prime}, l\right)\right| R_{E l}\right\rangle, \quad l^{\prime}=l \pm 1,
$$

while the remaining four terms include the integrated terms $J^{m m^{\prime}}$ (A.4) with $m, m^{\prime}=0,1$ :

$$
\begin{gathered}
M_{l l}^{l+1}\left(E^{\prime}, E, \mathscr{E}\right)=\frac{p}{E-\mathscr{E} \mid} \frac{l+1-i a}{|l+1-i a|} d_{l l+1}\left(E^{\prime}, E\right) \\
-\frac{p^{\prime}}{E^{\prime}-\mathscr{E}} \frac{l+1-i a^{\prime}}{\left|l+1-i a^{\prime}\right|} d_{l+1}\left(E^{\prime}, E\right) \\
+\frac{2^{2 l+2} Z^{2}\left(p p^{\prime}\right)}{[(2 l+3) !]^{2}} C_{E l} C_{E^{\prime} l}\left[\left(l+2+i a^{\prime}\right)(l+2+i a) J^{00}\right. \\
+\left(l+2+i a^{\prime}\right)(l+1-i a) J^{01} \\
+\left(l+1-i a^{\prime}\right)(l+2+i a) J^{10}
\end{gathered}
$$




$$
\begin{aligned}
& \left.+\left(l+1-i a^{\prime}\right)(l+1-i a) J^{11}\right], \\
& M_{l+2 l+2}^{l+1}\left(E^{\prime}, E, \mathscr{E}\right)=-\frac{p}{E-\mathscr{E}} \frac{|l+2-i a|}{l+2-i a} d_{l+2 l+1}\left(E^{\prime}, E\right) \\
& +\frac{p^{\prime}}{E^{\prime}-\mathscr{E}} \frac{\left|l+2-i a^{\prime}\right|}{l+2-i a^{\prime}} d_{l+1 l+2}\left(E^{\prime}, E\right) \\
& +\frac{2^{2 l+2} Z^{2}\left(p p^{\prime}\right)^{l}}{[(2 l+3) !]^{2}(l+2-i a)\left(l+2-i a^{\prime}\right)} \\
& \times C_{E l+2} C_{E^{\prime} l+2}\left[J^{00}-J^{01}-J^{10}+J^{11}\right], \\
& M_{l+2 l}^{l+1}\left(E^{\prime}, E, \mathscr{E}\right)=\frac{p}{E-\mathscr{E}} \frac{l+1-i a}{|l+1-i a|} d_{l+2 l+1}\left(E^{\prime}, E\right) \\
& +\frac{p^{\prime}}{E^{\prime}-\mathscr{E}} \frac{\left|l+2-i a^{\prime}\right|}{l+2-i a^{\prime}} d_{l+1 l}\left(E^{\prime}, E\right) \\
& +\frac{2^{2 l+2} Z^{2}\left(p p^{\prime}\right)}{[(2 l+3) !]^{2}\left(l+2-i a^{\prime}\right)} \\
& \times C_{E l} C_{E l+2}\left[(l+2+i a) J^{00}+(l+1-i a) J^{01}\right. \\
& \left.-(l+2+i a) J^{10}-(l+1-i a) J^{11}\right] .
\end{aligned}
$$

Here, $a=Z / p$ is the standard Coulomb parameter, and $C_{E l}$ is the normalization factor for the continuum state $R_{E l}(r)$ :

$$
C_{E l}=\sqrt{\frac{2 p}{\pi}} \exp (\pi a / 2)|\Gamma(l+1-i a)|
$$

The MEs $d_{l l}$ satisfy the symmetry relations

$$
\begin{aligned}
d_{l l+1}\left(E^{\prime}, E\right) & =-d_{l+1 l}\left(E, E^{\prime}\right), \\
d_{l+2 l+1}\left(E^{\prime}, E\right) & =-d_{l+1 l+2}\left(E, E^{\prime}\right)
\end{aligned}
$$

and can be expressed explicitly (see Appendix A) in terms of the functions ${ }_{2} F_{1}$ (cf. the expression for the ME for a single-photon transition with the interaction operator in the "form of length" [55]):

$$
\begin{gathered}
d_{l+1 l}\left(E^{\prime}, E\right)=-\frac{2^{2 l+2} Z p^{l+1} p^{l}}{[(2 l+3) !]^{2}} \\
\times C_{E^{\prime} l+1} C_{E l}\left[(l+2+i a) I^{10}+(l+1-i a) I^{11}\right], \\
d_{l+1 l+2}\left(E^{\prime}, E\right)=-\frac{2^{2 l+2} Z p^{l+1} p^{l}}{[(2 l+3) !]^{2}(l+2-i a)} \\
\times C_{E^{\prime} l+1} C_{E l+2}\left(I^{10}-I^{11}\right) .
\end{gathered}
$$

Here,

$$
\begin{gathered}
I^{m^{\prime} m}\left(E^{\prime}, E\right)=(-1)^{l}(2 l+3) ! \\
\times \frac{\left(p-p^{\prime}+i 0\right)^{-l-1-m^{\prime}+i a^{\prime}}\left(p^{\prime}-p+i 0\right)^{-l-1-m+i a}}{\left(p+p^{\prime}\right)^{2+i a^{\prime}+i a-m^{\prime}-m}} \\
\times{ }_{2} F_{1}\left(l+1+m^{\prime}-i a^{\prime}, l+1+m-i a, 2 l+4, \lambda_{0}\right),
\end{gathered}
$$

where $\lambda_{0}=-4 p p^{\prime} /\left(p-p^{\prime}\right)^{2}$.

Expression (A.4) for $J^{m m^{\prime}}$ can be written only in the form of a one-dimensional integral of the function ${ }_{2} F_{1}$ with the same parameters as in Eq. (42):

$$
\begin{gathered}
J^{m^{\prime} m}(E, E, \mathscr{E})=2^{2 l+4}(2 l+3) ! v^{2 l+5} \int_{0}^{1} d t \\
\times \frac{t^{l+1-Z v}{ }_{2} F_{1}\left(l+1+m^{\prime}-i a^{\prime}, l+1+m-i a, 2 l+4 ; \lambda\right)}{A^{l+1+m-i a} B^{l+1+m^{\prime}-i a^{\prime}} C^{2-m^{\prime}-m+i a^{\prime}+i a}},
\end{gathered}
$$

where $v=1 / \sqrt{-2 \mathscr{E}}, \lambda=\left(16 p p^{\prime}|v|^{2} t\right) / A B$, and

$$
\begin{gathered}
A=(1-p|v|+i 0)\left(1+p^{\prime}|v|+i 0\right) \\
-t(1+p|v|-i 0)\left(1-p^{\prime}|v|-i 0\right), \\
B=(1+p|v|+i 0)\left(1-p^{\prime}|v|+i 0\right) \\
-t(1-p|v|-i 0)\left(1+p^{\prime}|v|-i 0\right), \\
C=(1+p|v|+i 0)\left(1+p^{\prime}|v|+i 0\right) \\
-t(1-p|v|-i 0)\left(1-p^{\prime}|v|-i 0\right)
\end{gathered}
$$

Formulas (43) and (44) are written under the assumption that the energy of the intermediate state is positive: $\mathscr{E}>0$. If $\mathscr{E}<0$ (this case is realized in elastic scattering with reemission of a photon with $\omega>E$ ), the parameter $v=1 / \sqrt{-2 \mathscr{E}}$ is real-valued and the substitution $|v| \longrightarrow$ $-i v$ must be carried out in Eqs. (43) and (44). Infinitely small corrections $\pm i 0$ in Eqs. (42) and (44), which determine the rules for raising negative quantities to a power, appear as a result of regularization of integrals (A.3) and (A.4) with oscillating functions. It can be seen that expressions (38)-(40) contain two types of terms: the hypergeometric functions ${ }_{2} F_{1}$ and integrals of ${ }_{2} F_{1}$. Relatively simple "integrated" terms make a dominating contribution to $M_{l^{\prime} l}^{L}(E, E, \mathscr{E})$ in the domain of the variables (see Section 5) and contain, in particular, the Born limit since the terms with integrals $J^{m^{\prime} m}$ have an extra factor $Z$.

In spite of the cumbersome form typical of analytic calculations with Coulomb functions of the continuum, formulas (38)-(40) and (43) cannot apparently be sim- 
plified further and are the simplest expressions generalizing Coulomb matrix elements of the type (41), (42) for single-photon bremsstrahlung processes to the case of two-photon free-free transitions. At the same time, the analytic expressions for the MEs for bound-bound and bound-free transitions following from them for analytic continuations in $E$ and $E^{\prime}$ can be simplified and reduced to two-photon Gordon formulas [49], which are free of integrations. The corresponding transformations can be found in Appendix B.

\section{ELIMINATION OF SINGULARITIES IN THE AMPLITUDE \\ OF ELASTIC TWO-PHOTON TRANSITIONS}

Elastic two-photon transitions in the continuous spectrum require special analysis in view of the convergence of the radial MEs of dipole transitions between the states of the continuum with identical energies even in the single-photon case. The situation in two-photon transitions is analogous: it can be seen from expressions (42) and (43) that all MEs $M_{l^{\prime} l}^{L}$ diverge for $E^{\prime} \longrightarrow E\left(p^{\prime} \longrightarrow p\right)$. The reason for the divergence can be easily grasped if we consider the asymptotic form of the radial Coulomb Green function integrated with the wave function of the continuum:

$$
\begin{gathered}
\int_{0}^{\infty} d r^{\prime} r^{\prime 2} g_{L}\left(\mathscr{E} ; r, r^{\prime}\right)\left(\frac{d}{d r^{\prime}}+\frac{A}{r^{\prime}}\right) R_{E l}\left(r^{\prime}\right) \underset{r \rightarrow \infty}{\longrightarrow} C_{1} r^{Z v-1} e^{-r / v} \\
+\frac{C_{2}}{r} \cos \left(p r+\frac{Z}{p} \ln (2 p r)-\frac{\pi}{2} l+\delta_{l}(p)\right),
\end{gathered}
$$

where $A, C_{1}$, and $C_{2}$ are constants. The presence of the second term oscillating with the same frequency as the wave function $R_{E l}(r)$ in the asymptotic form is responsible for the divergence of $M_{l^{\prime} l}^{L}\left(E^{\prime}, E, \mathscr{E}\right)$ for $E^{\prime} \longrightarrow E$ both in the case of the Coulomb potential and for $Z=0$. Since the cross sections of elastic processes are finite, the singularities in $M_{l^{\prime} l}^{L}$ must be compensated in the calculation of the limit $E^{\prime} \longrightarrow E$ in the sum

$$
M\left(\mathbf{e}^{*}, \mathbf{e}, E+\omega\right)+M\left(\mathbf{e}, \mathbf{e}^{*}, E-\omega^{\prime}\right),
$$

defining the total amplitude $f_{2}$ of the transition in relation (12). The first term in expression (45) corresponds to the absorption of a photon followed by its emission (see Fig. 1a), while the second term corresponds to the reverse process (see Fig. 1b). The quantities $\omega$ and $\omega^{\prime}$ in sum (45) are connected through the relation $\omega-\omega^{\prime}=E^{\prime}-E$.

It follows from expression (19) for $M\left(\mathbf{e}^{*}, \mathbf{e}, \mathscr{E}\right)$ that the polarization-angular parameter $Q_{4}$ reverses its sign upon the transposition of $\mathbf{e}$ and $\mathbf{e}^{*}$, while the signs of the remaining $Q_{i}$ remain unchanged. It follows hence that singularities for $E^{\prime} \longrightarrow E$ must be compensated in the combinations of MEs,

$$
\begin{gathered}
S_{l^{\prime} l}^{L}=\lim _{E^{\prime} \rightarrow E}\left[M_{l^{\prime} l}^{L}\left(E^{\prime}, E, E+\omega\right)\right. \\
\left.+M_{l^{\prime} l}^{L}\left(E^{\prime}, E, E-\omega^{\prime}\right)\right], \\
R_{l}(\mathscr{E})=\lim _{E^{\prime} \rightarrow E}\left[M_{l l}^{l-1}\left(E^{\prime}, E, \mathscr{E}\right)-M_{l l}^{l+1}\left(E^{\prime}, E, \mathscr{E}\right)\right],
\end{gathered}
$$

through which we can express the parameters $Q_{i}^{\mathrm{el}}$ of elastic transition in formula (34):

$$
\begin{gathered}
Q_{1}^{\mathrm{el}}=-\sum_{l=2}^{\infty}\left[\frac{1}{2 l+3}\left(\mathscr{S}_{l l}^{l+1}+\mathscr{S}_{l+2 l}^{l+1}\right)\right. \\
\left.+\frac{1}{2 l-1}\left(\mathscr{Y}_{l l}^{l-1}+\mathscr{Y}_{l-2 l}^{l-1}\right)\right] P_{l}^{(2)}(x), \\
Q_{2}^{\mathrm{el}}=\sum_{l=1}^{\infty}\left[\frac{1}{2 l-1} \mathscr{Y}_{l l}^{l-1}+\frac{1}{2 l+3} \mathscr{S}_{l l}^{l+1}\right] P_{l}^{(1)}(x) \\
+2 \sum_{l=2}^{\infty}\left[\left(\frac{1}{2 l-1} \mathscr{S}_{l l}^{l-1}+\frac{1}{2 l+3} \mathscr{S}_{l l}^{l+1}\right) x\right. \\
\left.\frac{1}{2 l+1}\left(\mathscr{S}_{l-1}^{l}+1+\mathscr{S}_{l+1}^{l}\right)\right] P_{l}^{(2)}(x), \\
Q_{3}^{\mathrm{el}}=\sum_{l=1}^{\infty}\left[\mathscr{R}_{l}(E+\omega)-\mathscr{R}_{l}(E-\omega)\right] P_{l}^{(1)}(x), \\
Q_{4}^{\mathrm{el}}=\frac{1}{3} \sum_{l=0}^{\infty}\left[l \mathscr{S}_{l l}^{l-1}+(l+1) \mathscr{S}_{l l}^{l+1}\right] P_{l}(x),
\end{gathered}
$$

where

$$
\begin{gathered}
\mathscr{S}_{l^{\prime} l}^{L}=\left(2 \pi^{2} / p\right) \exp \left[i\left(\delta_{l^{\prime}}(p)+\delta_{l}(p)\right)\right] \mathscr{S}_{l^{\prime} l}^{L}, \\
\mathscr{R}_{l}(\mathscr{E})=\left(\pi^{2} / p\right) \exp \left[2 i \delta_{l}(p)\right] R_{l}(\mathscr{E}) .
\end{gathered}
$$

In order to verify the compensation of the divergences and to find the limits of expressions (46) and (47), we must explicitly separate the diverging and finite parts of the ME $M_{l^{\prime} l}^{L}$. We will illustrate the corresponding results using analytic expressions (38)-(40) for the Coulomb potential. This can easily be done for the integrated terms through the known asymptotic expansion of function ${ }_{2} F_{1}$ in inverse powers of argument [56]. The separation of singularities from $J^{m^{\prime} m}$ in expressions (38)-(40) requires more complex transformations. It should be noted above all (see Eq. (43)) that integrals $J^{00}$ and $J^{11}$ are finite for $E^{\prime}=E$, while $J^{01}$ and $J^{10}$ diverge as $\ln \left(E^{\prime}-E\right)$. The presence of the logarithmic singularity alone allows us to set $E^{\prime}=E$ in the coefficients of the integrated terms in expressions (38)-(40) 
everywhere except in factors $A$ and $B$. Using the technique for separating singularities described in [13], we can present the diagonal MEs in the form

$$
\begin{aligned}
& M_{l+2 l+2}^{l+1}\left(E^{\prime}, E, \mathscr{E}\right)=-\frac{a}{\pi} \frac{1}{E-\mathscr{E}}\left(\ln \left(\frac{p-p^{\prime}}{2 p}\right)^{2}-2 \psi(1)\right) \\
& +\frac{1}{E-\mathscr{E}} \frac{a}{\pi(l+2-i a)}\left(\frac{2 \mathscr{E}}{E-\mathscr{E}}+i a \psi(l+1-i a)\right. \\
& +i a \psi(l+2-i a)+2 i a \psi(l+3+i a) \\
& -2(l+2+i a) \operatorname{Re} \psi(l+2-i a)) \\
& +Z^{2} \frac{2^{2 l+2} p^{2 l}}{((2 l+3) !)^{2}} \frac{C_{E l+2}^{2}(l+2-i a)^{2}}{\left(J^{00}+J^{11}-K\right],} \\
& M_{l l}^{l+1}\left(E^{\prime}, E, \mathscr{E}\right)=-\frac{a}{\pi} \frac{1}{E-\mathscr{E}}\left(\ln \left[\left(\frac{p-p^{\prime}}{2 p}\right)^{2}\right]-2 \psi(1)\right) \\
& +\frac{1}{E-\mathscr{E}} \frac{a}{\pi(l+1+i a)}\left(-\frac{2 \mathscr{E}}{E-\mathscr{E}}+\frac{i a}{l+1-i a}\right. \\
& +\frac{i a}{l+2+i a}-i a \psi(l+1-i a)-i a \psi(l+2-i a) \\
& +2 i a \psi(l+3+i a)-2(l+1-i a) \operatorname{Re} \psi(l+2-i a)) \\
& \left.+(l+1-i a)^{2} J^{11}+(l+1-i a)(l+2+i a) K\right],
\end{aligned}
$$

where $K$ is the regular part of the sum $J^{01}+J^{10}$,

$$
\begin{aligned}
& K=-\frac{2^{2 l+4}(2 l+3) ! v^{2 l+5}}{\left(1-p^{2}|v|^{2}\right)(l+1-i a)} \int_{0}^{1} d t \frac{t^{l-Z v}}{A^{2 l+2-2 i a} C^{1+2 i a}} \\
& \times\left[\left(i a(1-p|v|)+\frac{t}{C}(1+2 i a)(1-p|v|)^{2}\right)\right. \\
& \quad \times{ }_{2} F_{1}(l+1-i a, l+1-i a, 2 l+4, \lambda) \\
& \left.+(l+1-i a)_{2} F_{1}(l+1-i a, l+2-i a, 2 l+4, \lambda)\right],
\end{aligned}
$$

and $\psi(x)=(d / d x) \ln \Gamma(x)$ is the psi function. Integrals $J^{m m}$ in expressions (49) and (50) are defined by formula (43) with $p^{\prime}=p$. In this case,

$$
\begin{gathered}
A=B=\left(1-p^{2}|v|^{2}\right)(1-t)+i 0 \\
C=(1+p|v|)^{2}-t(1-p|v|)^{2}, \quad \lambda=\frac{16 p^{2}|v|^{2} t}{A^{2}} .
\end{gathered}
$$

Considering that $E-\mathscr{E}=-\omega$ for the diagram presented in Fig. 1a and $E-\mathscr{E}=\omega^{\prime}$ for the diagram in Fig. 1b and that the singular terms in expressions (49) and (50) are identical and independent of $l$, we can easily see that the divergences in the calculation of $R_{l}(\mathscr{E})$ in expression (47) and in the diagonal elements $S_{l l}^{L}$ in expression (46) are compensated and the final results obviously follow from expressions (49) and (50).

The nondiagonal MEs contain, in addition to the logarithmic singularity, a power singularity; consequently, their singular part has the form

$$
\begin{gathered}
{\left[M_{l+2 l}^{l+1}\left(E^{\prime}, E, \mathscr{E}\right)\right]_{\text {sing }}=\frac{a}{\pi|l+1-i a||l+2+i a|}} \\
\quad \times\left(\frac{l+1-i a}{E-\mathscr{E}}+\frac{l+2+i a}{E^{\prime}-\mathscr{E}}\right) \frac{p}{p-p^{\prime}} \\
+\left(\frac{C(E, l)}{E-\mathscr{E}}+\frac{C\left(E^{\prime}, l\right)}{E^{\prime}-\mathscr{E}}\right) \ln \left[\left(\frac{p-p^{\prime}}{2 p}\right)^{2}\right] .
\end{gathered}
$$

When we evaluate the limit $E^{\prime} \longrightarrow E$ in sum (46) with $l^{\prime}=l+2$, the terms with the logarithmic singularities vanish, while the terms with the power singularities make a finite contribution. As a result, the final expression for $S_{l+2 l}^{l+1}$ assumes the form

$$
\begin{gathered}
S_{l+2 l}^{l+1}=\frac{Z p(1+2 i a)}{\pi|l+1-i a||l+2+i a|} \frac{1}{\omega^{2}} \\
+\frac{Z^{2} 2^{2 l+2} p^{2 l}}{[(2 l+3) !]^{2}(l+2-i a)} \\
\times\left[(l+2+i a)\left(J^{00}(E+\omega)+J^{00}(E-\omega)\right)\right. \\
-(l+1-i a)\left(J^{11}(E+\omega)+J^{11}(E-\omega)\right) \\
-(1 / 2+i a)(K(E+\omega)+K(E-\omega))] .
\end{gathered}
$$

The functions ${ }_{2} F_{1}$ in the integrands of the integrals $J^{m m^{\prime}}$ and $K$ appearing in expressions (49), (50), and (54) have a branching point for $\lambda=1$, lying on the integration contour. The choice of the required analytic branch is determined by the imaginary correction in expression (52) for A. Expressions (49), (50), and (54) completely determine the amplitude $f_{2}$ in expression (12) and the cross section (34) of elastic two-photon transition in the Coulomb field.

\section{ASYMPTOTIC ANALYSIS OF THE AMPLITUDES OF INELASTIC TWO-PHOTON TRANSITIONS}

The partial-wave approach leads to expressions for parameters $Q_{i}\left(p, p^{\prime}, \theta\right)$ only in the form of series in Legendre polynomials, and the radial MEs appearing in these series can be calculated analytically only in the Coulomb case. Nevertheless, we can derive quite simple closed expressions for $Q_{i}$ and transition amplitudes 
in potential $U(r)$ of the general form for the number of limiting ranges of the parameters of the problem, which will be considered below.

\subsection{Low-Frequency Limit $\left(\omega / p^{2} \ll 1\right)$}

Let us first consider first-order MEs $d_{l^{\prime} l}\left(E^{\prime}, E\right)(37)$. Since the oscillation frequencies of the wave functions of the initial and final states become close for $E^{\prime} \longrightarrow E$, the radial integral in formula (37) diverges at infinity. Replacing the wave functions by their asymptotic expressions for $r \longrightarrow \infty$,

$$
R_{E l} \longrightarrow \sqrt{\frac{2 p}{\pi}} \sin \left(p r-\frac{1}{2} \pi l+\delta_{l}(p)\right),
$$

and retaining in expression (37) only the principal terms in $1 / r$, we obtain

$$
\begin{gathered}
d_{l^{\prime} l}=-\frac{p}{\pi} \operatorname{Re}\left(\frac{\exp \left(-i \Delta_{l^{\prime} l}(E)\right)}{E^{\prime}-E+i 0}\right) \\
=p \sin \Delta_{l^{\prime} l}(E) \delta\left(E^{\prime}-E\right)+\frac{p}{\pi} \frac{\cos \Delta_{l^{\prime} l}(E)}{E^{\prime}-E},
\end{gathered}
$$

where $\Delta_{l^{\prime} l}(E)=\delta_{l}(p)-\delta_{l^{\prime}}(p)-(\pi / 2)\left(l-l^{\prime}\right)$. The presence of the $\delta$ function in the MEs of the free-free transitions and the method of determining of their asymptotic form are well known (see Section 21 in [57]). In real singlephoton transitions between the states of the continuum (with $E^{\prime} \neq E$ ), the singular term is omitted; however, in compound MEs, integration is carried out with respect to the energies of virtual states and the $\delta$ term in $d_{l^{\prime} l}$ plays a significant role. The problems associated with its inclusion in numerical and analytic calculations have been repeatedly discussed in the literature $[14$, 58-62].

Let us now consider the low-frequency limit of the second-order MEs $M_{l^{\prime} l}^{L}\left(E^{\prime}, E, \mathscr{E}\right)$ with $\mathscr{E}=E \pm \omega$ and $E^{\prime}=\mathscr{E} \pm \omega^{\prime}$. Using the spectral expansion for the Green function in relations (21), we can represent $M_{l^{\prime} l}^{L}$ in the form

$$
\begin{gathered}
M_{l^{\prime} l}^{L}\left(E^{\prime}, E, \mathscr{E}\right)=-\sum_{n} \frac{d_{l^{\prime} L}\left(E^{\prime}, E_{n}\right) d_{L l}\left(E_{n}, E\right)}{E_{n}-\mathscr{E}} \\
-\int d \epsilon \frac{d_{l^{\prime} L}\left(E^{\prime}, \epsilon\right) d_{L l}(\epsilon, E)}{\epsilon-\mathscr{E}-i 0},
\end{gathered}
$$

where summation is carried out over the states of the discrete spectrum, while integration is carried out over the states of the continuous spectrum of the Hamiltonian with potential $U(r)$. In the low-frequency range, the two terms in Eq. (57) have different values; we can easily verify that the sum over the discrete spectrum gives a finite result for $\omega, \omega^{\prime} \longrightarrow 0$, while the integral is of the order of $1 / \omega \omega$ ' in accordance with the general nature of the frequency dependence of the amplitude of scattering accompanied by the emission of soft photons [3]. It turns out that the main contribution to the integral in expression (57), leading to the above-mentioned singularity for $\omega, \omega^{\prime} \longrightarrow 0$, is associated with $\delta$-shaped terms in the integrand function, whose presence follows from relation (56) and from the well-known relation

$$
\frac{1}{\epsilon-\mathscr{E}-i 0}=\mathrm{V} \cdot \mathrm{p} \cdot \frac{1}{\epsilon-\mathscr{E}}+i \pi \delta(\epsilon-\mathscr{E}) \text {. }
$$

Omitting the sum over the discrete spectrum in expression (57) and taking into account only the contribution from the points $\epsilon=E, E^{\prime}, \mathscr{E}$ in the integral, we obtain the following intermediate expression for $M_{l^{\prime} l}^{L}$ :

$$
\begin{aligned}
M_{l^{\prime} l}^{L} & =p^{\prime} \sin \Delta_{l^{\prime} L}\left(E^{\prime}\right) \frac{1}{E^{1}-\mathscr{E}} d_{L l}\left(E^{\prime}, E\right) \\
+ & p \sin \Delta_{L l}(E) \frac{1}{E-\mathscr{E}} d_{l^{\prime} L}\left(E^{\prime}, E\right) \\
& -i \pi d_{l^{\prime} L}\left(E^{\prime}, \mathscr{E}\right) d_{L l}(\mathscr{E}, E) .
\end{aligned}
$$

Replacing the MEs $d_{l_{2} l_{1}}\left(E_{2}, E_{1}\right)$ by their limiting expressions for $E_{2} \longrightarrow E_{1}$ (second term in Eq. (56)) and retaining only the principal term in the asymptotic form for $\omega, \omega^{\prime} \longrightarrow 0$, we obtain

$$
\begin{gathered}
M_{l^{\prime} l}^{L}=\frac{p^{2}}{\pi} \frac{\sin \Delta_{l^{\prime} L}(E) \cos \Delta_{L l}(E)}{\left(E^{\prime}-\mathscr{E}\right)\left(E^{\prime}-E\right)} \\
+\frac{p^{2}}{\pi} \frac{\cos \Delta_{l^{\prime} L}(E) \sin \Delta_{L l}(E)}{(E-\mathscr{E})\left(E^{\prime}-E\right)} \\
-i \frac{p^{2}}{\pi} \frac{\cos \Delta_{l^{\prime} L}(E) \cos \Delta_{L l}(E)}{\left(E^{\prime}-\mathscr{E}\right)(\mathscr{E}-E)} .
\end{gathered}
$$

In the low-frequency limit, we cannot only simplify partial MEs, but also sum the series in relations (20) in the general form for parameters $Q_{i}$. Let us consider for definiteness the process of induced $2 \mathrm{BrS}: \mathbf{e}_{1}=\mathbf{e}_{2}=\mathbf{e}^{*}$, $\mathscr{E}=E-\omega, E^{\prime}=E-2 \omega$. Then relation (60) leads to the following expressions for $\mathcal{M}_{l^{\prime} l}^{l^{\prime \prime}}$ :

$$
\begin{gathered}
\mathcal{M}_{l l}^{l+1}=\frac{\pi p}{i \omega^{2}}\left(e^{2 i \delta_{l}(p)}-e^{2 i \delta_{l+1}(p)}\right), \\
\mathcal{M}_{l l}^{l-1}=\frac{\pi p}{i \omega^{2}}\left(e^{2 i \delta_{l}(p)}-e^{2 i \delta_{l-1}(p)}\right), \\
\mathcal{M}_{l+2 l}^{l+1}=\frac{\pi p}{2 i \omega^{2}}\left(2 e^{2 i \delta_{l+1}(p)}-e^{2 i \delta_{l+2}(p)}-e^{2 i \delta_{l}(p)}\right) .
\end{gathered}
$$

Substituting expressions (61) into (20) and using the recurrence relation for the derivatives of the Legendre polynomial [56], we present parameter $Q_{1}$ in the form

$$
Q_{1}=\frac{\pi p}{2 i \omega^{2}} \sum_{l=0}^{\infty}(2 l+1) P_{l}(x) e^{2 i \delta_{l}(p)} .
$$


Taking now into account the well-known relation for the amplitude $f_{0}$ of elastic scattering in the field $U(r)$ [57],

$$
f_{0}(\theta)=\frac{1}{2 i p} \sum_{l}(2 l+1) P_{l}(\cos \theta) e^{2 i \delta_{l}(p)},
$$

we express $Q_{1}$ in terms of $f_{0}$ :

$$
Q_{1}=\frac{\pi p^{2}}{\omega^{2}} f_{0} .
$$

Similarly, we can express the remaining parameters $Q_{i}$ in relations (20) in terms of $f_{0}$ with the help of appropriate recurrence relations for $P_{k}^{(m)}(x)$ and find that

$$
Q_{2}=Q_{1}, \quad Q_{3}=-2 Q_{1}, \quad Q_{5}=\frac{2}{3}(1-\cos \theta) Q_{1} .
$$

As a result, we obtain the following expression for the 2BrS amplitude $M\left(\mathbf{e}^{*}, \mathbf{e}^{*}, E-\omega\right)$ in expression (19),

$$
M=\frac{\pi p^{2}}{\omega^{2}} f_{0}\left(\mathbf{e}^{*} \cdot\left(\mathbf{n}-\mathbf{n}^{\prime}\right)\right)^{2},
$$

and the low-frequency asymptotic form of the cross section (see Eq. (9)) has the form

$$
d \sigma=\frac{F^{4}}{2^{6} \omega^{8}}\left|\mathbf{e} \cdot\left(\mathbf{p}-\mathbf{p}^{\prime}\right)\right|^{4} d \sigma_{0} .
$$

The same result follows from the Kroll-Watson formula (expression (6) with $d \sigma_{B} \longrightarrow d \sigma_{0}=\left|f_{0}\right|^{2} d \Omega$ ) if we pass to a low field strength $F$ in it.

Let us write for reference the low-frequency asymptotic expressions for $2 \mathrm{BrS}$ partial MEs (61) in the Coulomb field. In this case,

$$
e^{i \delta_{l}(p)}=\frac{\Gamma(l+1-i a)}{|\Gamma(l+1-i a)|},
$$

so that we obtain from relations (61)

$$
\begin{gathered}
M_{l l}^{l+1}=\frac{Z p}{\pi \omega^{2}} \frac{l+1-i a}{(l+1)^{2}+a^{2}}, \\
M_{l+2 l+2}^{l+1}=-\frac{Z p}{\pi \omega^{2}} \frac{l+2+i a}{(l+2)^{2}+a^{2}}, \\
M_{l+2 l}^{l+1}=-\frac{Z p}{\pi \omega^{2}} \frac{1 / 2+i a}{|(l+1-i a)(l+2-i a)|} .
\end{gathered}
$$

We considered above the arbitrary potential $U(r)$ (the general method of estimating ME in the low-frequency region was used earlier [62]); however, the behavior of Coulomb amplitudes in the low-frequency range can also be analyzed proceeding from exact expressions derived in Section 3. Expanding the functions ${ }_{2} F_{1}$ in the expression for $M_{l^{\prime} l}^{L}$ in inverse powers of the argument and retaining the principal term in $1 / \omega$, we arrive at results exactly coinciding with relations (66).
It turns out that the contribution to the main term of the asymptotic form comes only from the integrated terms. Formula (59), taking into account the contribution from the $\delta$-type singularities in the compound ME only and appearing in our calculations as an intermediate expression, was derived in an analogous way by Korol [14] and was used for approximate evaluation of Coulomb amplitudes in the entire range of variables. A comparison with the available data from the literature [11, 12] shows that formula (59), which is simple for calculations, successfully reproduces the results of exact calculations of the spontaneous $2 \mathrm{BrS}$ cross section. The existence of exact analytic expressions for Coulomb amplitudes makes it possible to establish the source of such good agreement. Comparing relation (59) with (38)-(40) and taking into account relations (65), we can easily verify that the real part in Korol's formula (59) exactly coincides with the real part of the integrated terms in $M_{l^{\prime} l}^{L}$. At the same time, the imaginary part in relation (59) is exact. Thus, formula (59) disregards only the real part of the integral terms in (38)-(40). It was mentioned above that the integrated terms make the main contribution to the amplitude in the Born and low-frequency region. The first term in the asymptotic form of the integral part of $M_{l^{\prime} l}^{L}$ in the Born region, as well as in the low-frequency range, turns out to be purely imaginary so that correction appears in formula (59) only in the next order and has the relative value

$$
\frac{a^{2} \omega^{2}}{E^{2}}\left[\ln ^{2}\left(\frac{\omega}{E}\right)+C \ln \left(\frac{\omega}{E}\right)+C^{\prime}\right]
$$

where $C$ and $C^{\prime}$ are constants. This estimate determines the accuracy of approximation of formula (59) for the Coulomb field.

It should be noted that the low-frequency KrollWatson approximation is inapplicable for small scattering angles, when the inclusion of only the principal term of expression (60) of the asymptotic form of the ME $M_{l^{\prime} l}^{L}$ for $\omega, \omega^{\prime} \longrightarrow 0$ is insufficient for estimating amplitudes $Q_{i}$. Since the estimation of corrections to expression (60) for the field $U(r)$ of the general form is complicated, we will illustrate this statement for the Coulomb scattering. In this case, the low-frequency asymptotic form (64) is given by

$$
\frac{d \sigma}{d \Omega}=\frac{1}{4 \pi^{2}}\left(\frac{F}{2 \omega}\right)^{4} \frac{p^{\prime}}{p} f_{\text {reg }}=B\left|\mathbf{e} \cdot\left(\mathbf{n}-\mathbf{n}^{\prime}\right)\right|^{4}
$$

where

$$
B=\frac{F^{4} Z^{2}}{2^{8} \omega^{8}} \frac{1}{\sin ^{4}(\theta / 2)} .
$$

Corrections of the next order in frequency are cumbersome and can be obtained from the exact formulas 
obtained in Section 3. In particular, in the next order in $\omega$, there appears the CD term

$$
\begin{gathered}
\frac{1}{4 \pi^{2}}\left(\frac{F}{2 \omega}\right)^{4} \frac{p^{\prime}}{p} \Delta_{C D} \\
=B \frac{2 a \omega}{p^{2}}(\ln y+b) \xi\left(\mathbf{k} \cdot\left[\mathbf{n} \times \mathbf{n}^{\prime}\right]\right)\left|\mathbf{e} \cdot\left(\mathbf{n}-\mathbf{n}^{\prime}\right)\right|^{2},
\end{gathered}
$$

where

$$
y=\frac{4 p p^{\prime}}{\left(p-p^{\prime}\right)^{2}} \sin ^{2} \frac{\theta}{2}
$$

and $b=b(a)$ is a smooth function of the momentum $p$. Obviously, the condition of applicability of the KrollWatson approximation is the fulfillment of the inequality

$$
\frac{a \omega}{p^{2}}\left|\left(\mathbf{k} \cdot\left[\mathbf{n} \times \mathbf{n}^{\prime}\right]\right)\right| \ll\left|\mathbf{e} \cdot\left(\mathbf{n}-\mathbf{n}^{\prime}\right)\right|^{2} .
$$

Since $\left|\mathbf{e} \cdot\left(\mathbf{n}-\mathbf{n}^{\prime}\right)\right|^{2} \sim \theta^{2}$ and $\left|\mathbf{n} \times \mathbf{n}^{\prime}\right| \sim \theta$ for $\theta \longrightarrow 0$, condition (70) is obviously violated for small scattering angles (the fulfillment of this condition naturally depends also on the azimuth angle). It should be noted that, since correction (69) depends on $\xi$, dichroism effects are significant in the $2 \mathrm{BrS}$ cross section even for small values of the ratio $\omega / p^{2}$ in this range of angles (although the CD effect obviously vanishes for $\theta=0)$.

\subsection{High-Frequency Limit $\left(Z / p \ll 1, \omega / E^{\prime} \gg 1\right.$, $\omega / E \sim 1)$ and Born Approximation}

Let a fast electron lose a considerable part of its energy during induced $2 \mathrm{BrS}$ so that the final state is not of the Born type. In this case, the replacement of the wave function of the initial state by the function of free motion,

$$
R_{E l}(r) \longrightarrow R_{E l}^{(0)}(r), \quad R_{E l}^{(0)}=\sqrt{\frac{2 p}{\pi}} j_{l}(p r),
$$

leads to the following results of the action of operators $D$ on the wave function:

$$
\begin{gathered}
D(l+1, l) R_{E l}^{(0)}=-p R_{E l+1}^{(0)}, \\
D(l+1, l+2) R_{E l+2}^{(0)}=p R_{E l+1}^{(0)} .
\end{gathered}
$$

Substituting now Green's function for a free electron for Green's function $g_{L}$ in relations (36) and using relations (72), we reduce $M_{l^{\prime} l}^{L}$ to the form

$$
M_{l^{\prime} l}^{L}=(-1)^{\left(l-l^{\prime}\right) / 2} \frac{2 E}{E-\mathscr{E}}\left\langle R_{E^{\prime} l^{\prime}} \mid R_{E l^{\prime}}^{(0)}\right\rangle .
$$

Evaluating the overlap integral $\left\langle R_{E^{\prime \prime}} \mid R_{E l^{\prime}}^{(0)}\right\rangle$ [63] and proceeding to the limit $E \longrightarrow \infty$, we obtain

$$
M_{l^{\prime} l}^{L}=(-1)^{\left(l-l^{\prime}\right) / 2} 2^{2 l^{\prime}+9 / 2} Z \frac{C_{E l^{\prime}}\left(l^{\prime}+1\right) ! p^{l^{\prime}}}{\sqrt{\pi}\left(2 l^{\prime}+2\right) ! p^{l^{\prime}+7 / 2}} .
$$

Comparing relations (72) with (A.1) and (A.2), we can easily establish that expression (74) in the Coulomb case corresponds to the inclusion in relations (38)-(40) of only the integrated term with $d_{l^{\prime} l+1}$ in which the wave function of the initial state is replaced by $R_{E l}^{(0)}$. A more detailed analysis of expressions (38)-(40) in the high-frequency range shows that the term with $d_{l^{\prime} l+1}$ makes the main contribution to the asymptotic form of $M_{l^{\prime} l}^{L}$ only for $L=l^{\prime}+1$, while all the terms with $M_{l+2 l}^{l+1}$ and $M_{l+2 l+2}^{l+1}$ (including the real part of the integral terms) are of the same order of magnitude for $L=l^{\prime}-1$. Thus, expression (74) is a correct high-frequency asymptotic form for the MEs $M_{l l}^{l+1}$ and $M_{l l+2}^{l+1}$ and differs from the correct result for $M_{l+2 l}^{l+1}$ and $M_{l+2 l+2}^{l+1}$ in the coefficient of $p^{-l^{\prime}-7 / 2}$.

It is interesting to note that an analogous situation also emerges in an analysis of single-photon transitions: substitution (71) in the MEs of photoionization,

$$
d_{l_{f} l_{i}}\left(E_{f}, E_{n_{i}}\right)=\left\langle R_{E_{f} l_{f}}\left|D\left(l_{f}, l_{i}\right)\right| R_{n_{i} l_{i}}\right\rangle,
$$

leads to a correct asymptotic form of MEs with $l_{f}=$ $l_{i}+1$ and gives an erroneous factor in the energy dependence for $l_{f}=l_{i}-1$ (this fact is mentioned in the monograph [55], although its origin is not discussed). In order to clarify the reason for such results, we consider the formation of the high-frequency asymptotic form of the photoionization MEs in the Born approximation:

$$
d_{l-1 l}^{(0)}\left(E_{f}, E_{n_{i}}\right)=\left\langle R_{E_{f} l-1}^{(0)}\left|\left(\frac{d}{d r}+\frac{l+1}{r}\right)\right| R_{n_{i} l}\right\rangle .
$$

The integral in this relation contains the rapidly oscillating (for $E_{f} \longrightarrow \infty$ ) spherical Bessel function $j_{l_{f}}(p r)$; consequently, the main contribution comes from the neighborhood of point $r=0$. Since $R_{n_{i} l} \sim N_{n_{i}} l^{l}$ for small $r$, it follows from expression (76) that

$$
\begin{gathered}
d_{l-1 l}\left(E_{f}, E_{n_{i}}\right) \\
=(2 l+1) N_{n_{i} l} \int_{0}^{\infty} r^{l+1} R_{E_{f} l-1}^{(0)}(r) d r .
\end{gathered}
$$


Using the well-known formula [63], we obtain the following expression for an integral of the form (77):

$$
\begin{gathered}
\int_{0}^{\infty} r^{l_{1}} R_{E l_{2}}^{0}(r) d r \\
=\frac{\Gamma\left(\frac{l_{2}+l_{1}+1}{2}\right)}{\Gamma\left(\frac{l_{2}-l_{1}+2}{2}\right)} 2^{l_{1}-1 / 2}(2 E)^{-\left(2 l_{1}+1\right) / 4} .
\end{gathered}
$$

For $l_{1}=l+1$ and $l_{2}=l-1$, the gamma function in the denominator of this expression becomes infinitely large; consequently, the principal term in the expansion of $R_{n_{i} l}$ in $r$ does not contribute to the asymptotic form. In order to evaluate the first nonvanishing term of the high-frequency asymptotic form of $d_{l-1 l}\left(E_{f}, E_{n_{i}}\right)$, we must not only continue the expansion of $R_{n_{i} l_{i}}$ in $r$, but also take into account the next terms in the expansion of the wave function $R_{E l}$ in $1 / E$, i.e., the correction to $R_{E l}^{(0)}$ in expressions (71), as well as the Coulomb correction to Green's function of a free electron for the second-order MEs. We omit here these calculations and only note that the main contribution to the asymptotic form of the total ME $M\left(\mathbf{e}^{*}, \mathbf{e}^{*}, E-\omega\right)$ in relation (7) comes from the partial amplitudes with $l^{\prime}=0$, whose high-frequency asymptotic form is given by formula (74) (it should be recalled that all partial amplitudes in the low-frequency region had the same order of magnitude in $\omega$ ).

In the Coulomb case, taking into account in relations (20) only the terms with $l^{\prime}=0$, which appear only in $Q_{1}$ and $Q_{5}$,

$$
Q_{1}=3 Q_{5}=2^{5} \pi Z e^{\pi a^{\prime} / 2} \Gamma\left(1-i a^{\prime}\right) p^{-4},
$$

we arrive at the following expression for the total amplitude:

$$
\begin{gathered}
M\left(\mathbf{e}^{*}, \mathbf{e}^{*}, E-\omega\right) \\
=2^{5} \pi Z e^{\pi a^{\prime} / 2} \Gamma\left(1-i a^{\prime}\right) p^{-4}\left(\mathbf{e}^{*} \cdot \mathbf{n}\right)^{2} .
\end{gathered}
$$

It should be noted that the quantitative agreement between the asymptotic and exact results is improved significantly if we calculate the asymptotic form retaining the exact normalization factor $C_{E l}$ in $M_{l^{\prime} l}^{L}$, which corresponds to the substitution $\sqrt{2 p / \pi} \longrightarrow C_{E l} / l$ ! in relations (71).

Since the high-frequency asymptotic form of $M_{l l}^{l+1}$ and $M_{l l+2}^{l+1}$ is determined by the integrated terms (see above), formula (59), taking into account these terms, exactly gives the correct limit (74) for these MEs in this region also. On the contrary, the high-frequency limit of formula (59) for $M_{l+2 l+1}^{l+1}$ and $M_{l+2 l+2}^{l+1}$ coincides with relation (74) and differs from the correct result.

If an electron remains fast in the final state also $\left(a^{\prime}=\right.$ $\left.Z / p^{\prime} \ll 1\right)$, we can apply the Born approximation. According to the results obtained by Bunkin and Fedorov [25] (see Eq. (6)), the induced 2BrS cross section in the Coulomb field in this case has the elementary form

$$
\begin{gathered}
\frac{d \sigma_{B}}{d \Omega}=\frac{F^{4} Z^{2}}{2^{4} \omega^{8}} \frac{\left.\mathbf{e} \cdot\left(\mathbf{p}-\mathbf{p}^{\prime}\right)\right|^{4}}{\left(\mathbf{p}-\mathbf{p}^{\prime}\right)^{4}} \frac{p^{\prime}}{p}, \\
p^{\prime 2}=p^{2}-4 \omega .
\end{gathered}
$$

This result can also be obtained directly from the exact results of partial-wave analysis. In the low-frequency region $\omega \ll p^{2}$ (although the frequency in this case may be significant as compared to the binding energy, $\omega \sim 1$ ), the partial Born series can be reduced to low-frequency series in which we must assume that $a \longrightarrow 0$ (see Subsection 5.1). For an arbitrary $\omega$, partial Coulomb MEs $M_{l^{\prime} l}^{L}$ are also simplified significantly in the Born region: first, the terms containing integrals in relations (38) - (40) are small for $a, a^{\prime} \ll 1$ since these terms contain an extra factor $Z / p$; second, the parameters of functions ${ }_{2} F_{1}$ become integral so that these function can be reduced to elementary functions. For example, the expression for $M_{l l}^{l+1}$ in the Born limit assumes the form

$$
\begin{gathered}
M_{l l}^{l+1}=Z \frac{(-1)^{l} 4^{l+1}(l !)^{2}}{(2 l+3) !} \frac{(l+1)\left(p p^{\prime}\right)^{l+1 / 2}}{\left(p-p^{\prime}\right)^{2 l+4}} \\
\times\left[(l+2) \frac{p^{2}+p^{\prime 2}}{\omega\left(p+p^{\prime}\right)^{2}} F_{1}\left(l+1, l+2,2 l+4 ; \lambda_{0}\right)\right. \\
\left.+4(l+1)_{2} F_{1}\left(l+2, l+2,2 l+4 ; \lambda_{0}\right)\right],
\end{gathered}
$$

where $\lambda_{0}=-4 p p^{\prime} /\left(p-p^{\prime}\right)^{2}$. Moreover, assuming that $a=$ $a^{\prime}=0$ in the integrand in formulas (38)-(40), we can calculate the second Born correction from the integrated terms in radial MEs in elementary form as well. However, the explicit expressions of these integrals and of functions ${ }_{2} F_{1}$ with integral parameters in terms of elementary (power and logarithmic) functions turn out to be cumbersome and become more and more involved with increasing $l$, which complicates the summation of partial series in $l$ in formulas (20). In the first Born approximation, such a summation can be carried out by using the expansion (see formulas (5.17.26) and (5.17.32) in [50])

$$
\frac{1}{\left(\mathbf{p}-\mathbf{p}^{\prime}\right)^{2}}=\sum_{l=0}^{\infty} a_{l}\left(p, p^{\prime}\right) P_{l}(\cos \theta),
$$



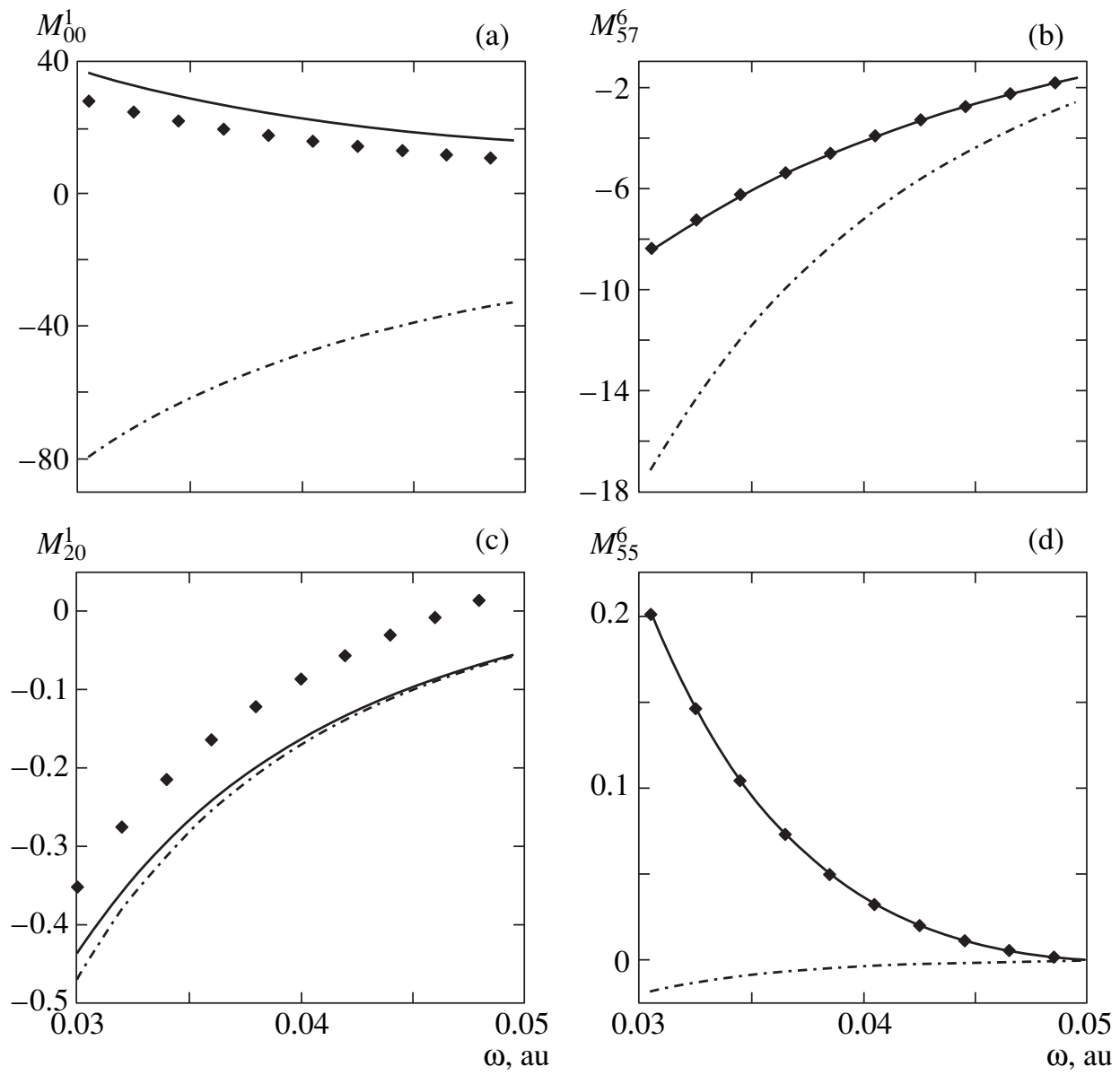

Fig. 3. Frequency dependences of the matrix elements of $2 \mathrm{BrS}$ for the initial electron energy $E=0.1$ (a, b) and 1.0 (c, d). Solid curves correspond to $\operatorname{Re} M$, dot-and-dash curves to $\operatorname{Im} M$, and rhombs to $\operatorname{Re} M$ in approximation (59).

$$
\begin{gathered}
a_{l}\left(p, p^{\prime}\right)=\frac{l !\left(p p^{\prime}\right)^{l}}{(1 / 2)_{l}\left(p-p^{\prime}\right)^{2 l+2}} \\
\times{ }_{2} F_{1}\left(l+1, l+1,2 l+2 ;-\frac{4 p p^{\prime}}{\left(p-p^{\prime}\right)^{2}}\right) .
\end{gathered}
$$

Using the recurrence relations for functions ${ }_{2} F_{1}$ and Legendre polynomials, we can verify that the summation of partial series for the amplitude in the Born limit results in expression (81).

\section{NUMERICAL RESULTS FOR THE COULOMB POTENTIAL AND DISCUSSION}

\subsection{Frequency and Energy Dependences of Radial Matrix Elements}

Since the cross sections of two-photon bremsstrahlung processes remain multiparametric functions even for fixed experimental geometry and photon polarization, it would be interesting to analyze qualitatively the dependence of MEs $M_{l^{\prime} l}^{L}\left(E^{\prime}, E, \mathscr{E}\right)$ on the frequency of photons and on the electron energy for various values the orbital angular momenta $l, L$, and $l$. The existence of exact formulas (38)-(40) for the Coulomb potential makes it possible to obtain quantitative results for MEs and cross sections in a wide range of these parameters since the problem is reduced to the evaluation of functions ${ }_{2} F_{1}$ and their integrals, which can easily be carried out using standard computer programs. It was shown in [64] that single-photon Coulomb MEs $d_{l^{\prime} l}\left(E^{\prime}, E\right)$ are positive monotonic functions of energy, which decrease monotonically with increasing $E^{\prime}$, say, for a fixed $E$ and $E^{\prime}>E$ and diverge for $E^{\prime} \longrightarrow E$ (in the presence of a non-Coulomb part in potential $U(r)$, the MEs may change their sign in a certain energy range depending on the value of the non-Coulomb correction to scattering phases [65]). In the two-photon case, the situation is complicated significantly due to the presence of an additional parameter, viz., the photon frequency $\omega$ (or the energy of an electron $\mathscr{E}=E \pm \omega$ in the virtual state), and in view of the complex nature of the MEs $M_{l^{\prime} l}^{L}$ (these matrix elements are real-valued only for $\mathscr{E}<0$, which corresponds to the re-emission of a photon with $\omega>E$ in the course of elastic scattering (see Fig. 1b)). 

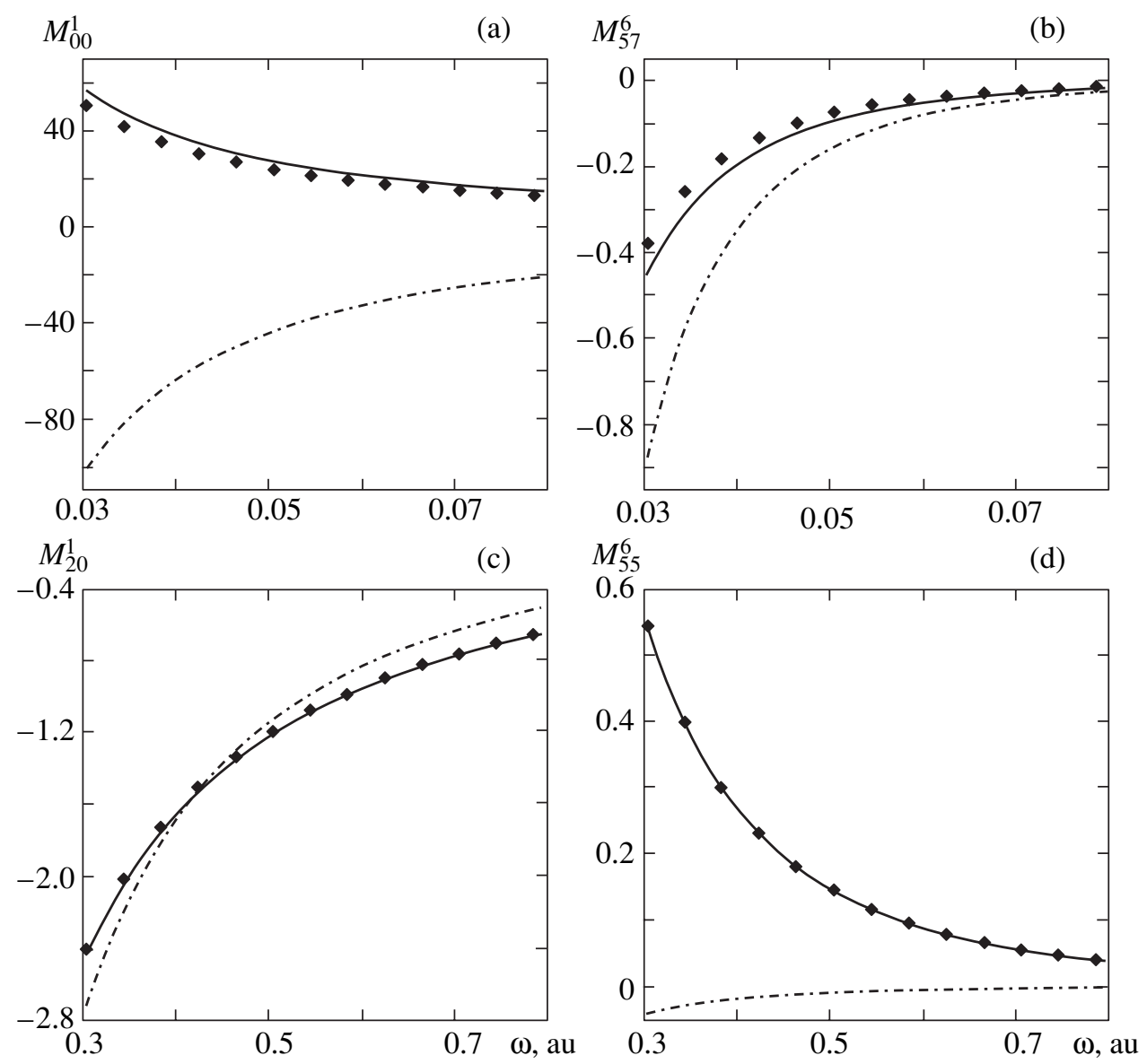

Fig. 4. The same as in Fig. 3 for the matrix elements of double bremsstrahlung absorption.

Since the imaginary parts of the MEs $M_{l^{\prime} l}^{L}$ can be reduced to the simple product of single-photon MEs (see formula (59)), it becomes especially interesting to establish the range of the parameters, in which the imaginary part makes a dominating contribution to two-photon MEs in view of the obvious radical simplification of the results.

It was mentioned above that, for a fixed $l$, the contribution to two-photon cross sections comes from two "diagonal" $\left(M_{l l}^{l+1}\right.$ and $\left.M_{l+1 l+1}^{l}\right)$ and two "nondiagonal" $\left(M_{l l+2}^{l+1}\right.$ and $\left.M_{l+2 l}^{l+1}\right)$ radial MEs. According to the results of calculations made in a wide range of $E, \omega$, and $l$, all these elements exhibit quite a universal behavior. The real and imaginary parts of all radial MEs (except $\operatorname{Re} M_{l l}^{l+1}$ ) are negative and decrease monotonically in absolute value with increasing frequency without reversing their signs (the imaginary part decreases at a higher rate than the real part). The signs of $\operatorname{Re} M_{l^{\prime} l}^{L}$ and $\operatorname{Im} M_{l^{\prime} l}^{L}$ are preserved upon a change in $l$. The absolute values of $\operatorname{Re} M_{l^{\prime} l}^{L}$ and $\operatorname{Im} M_{l^{\prime} l}^{L}$ for a given initial energy (outside the low-frequency region) decrease with increasing $l$ (the imaginary part decreases at a higher rate than the real part in this case also), the rate of their decrease with increasing frequency increasing with $l$. Figure 3 illustrates some examples of numerical calculations of the frequency dependence of radial MEs with $l=0$ and 5 for induced $2 \operatorname{BrS}\left(\mathscr{E}=E-\omega, E^{\prime}=E-2 \omega\right)$ for small $(E=0.1)$ and intermediate $(E=1.0)$ values of the initial energy $E$. It should be noted that all MEs assume finite values at the threshold frequency $\omega=E / 2$ $\left(E^{\prime}=0\right)$.

Figure 4 shows the frequency dependence of radial MEs of double bremsstrahlung absorption $(\mathscr{E}=E+\omega$, $\left.E^{\prime}=E+2 \omega\right)$ for the same values of $E$ and $l$ as in the case of $2 \mathrm{BrS}$ (see Fig. 3). It can be seen that the behavior of $M_{l^{\prime} l}^{L}$ for emission and absorption processes is qualitatively the same (monotonic decrease of $\operatorname{Re} M_{l^{\prime} l}^{L}$ and $\operatorname{Im} M_{l^{\prime} l}^{L}$ upon an increase in $\omega$ and angular momentum $l$ preserving fixed sign, and the rapid decrease in the imaginary part). The only difference is that there is no threshold limitation on frequency in the case of bremsstrahlung absorption, and the MEs decrease upon 
an increase in $\omega$, tending to an asymptotic form of type (74) (in a very far frequency range). For a given frequency $\omega$, the "diagonal" MEs for absorption are considerably larger than the corresponding MEs for $2 \mathrm{BrS}$, and $\left|M_{l l}^{l+1}\right|>\left|M_{l+1 l+1}^{l}\right|$. An interesting correspondence is observed between the numerical values of "nondiagonal" MEs for emission and absorption:

$$
M_{l^{\prime} l}^{L}(E+2 \omega, E+\omega, E) \leftrightharpoons M_{l l^{l}}^{L}(E-2 \omega, E-\omega, E) .
$$

In particular, the MEs in which the change in energy and in the orbital angular momentum occurs "in the same direction" have the largest magnitude in both cases in the major part of the frequency range. This corresponds to the well-known Bethe rule for single-photon transitions in a discrete spectrum [55]. However, for two-photon transitions in the continuous spectrum, this rule turns out to be not very stringent; it is violated in the low-frequency range, this region expanding with increasing angular momentum $l$.

The existence of exact results makes it possible to verify the correctness of approximations used for calculating $M_{l^{\prime} l}^{L}\left(E^{\prime}, \mathscr{E}, E\right)$. The simplest among them is the "pole" approximation, which consists in the inclusion of only the imaginary part of $M_{l^{\prime} l}^{L}$ in relation (57) (originating from the term with the $\delta$ function in Eq. (58)). In the conditions of applicability of perturbation theory in the wave field, the model of "significant states" [66, 67] in the theory of multiphoton transitions in a strong field can also be reduced to this approximation. Asymptotic estimates (66) and numerical calculations show that the imaginary part dominates in the low-frequency range for low initial electron energies (the accuracy of the pole approximation for "nondiagonal" MEs in this case is much higher than for "diagonal" MEs). In the pole approximation, a two-photon transition can be regarded as a "cascade" transition, i.e., a transition occurring only via an intermediate state of the continuum with energy $\mathscr{E}$, which corresponds to the factorization of the process amplitude:

$$
\begin{gathered}
M_{l^{\prime} l}^{L}\left(E^{\prime}, \mathscr{E}, E\right) \longrightarrow i \operatorname{Im} M_{l^{\prime} l}^{L} \\
=-i \pi\left\langle R_{E^{\prime} l^{\prime}}\left|D\left(l^{\prime}, L\right)\right| R_{\mathscr{E} L}\right\rangle\left\langle R_{\mathscr{E} L}|D(L, l)| R_{E l}\right\rangle .
\end{gathered}
$$

As the frequency and/or the electron energy increases, the contribution of the omitted real part of MEs increases. In the high-frequency region $\left(a \ll 1, \omega / E^{\prime} \gg 1\right.$, $\omega / E \sim 1$ for emission and $a^{\prime} \ll 1, \omega / E^{\prime} \sim 1, \omega / E \gg 1$ for absorption), the real part of $M_{l^{\prime} l}^{L}$ becomes dominating, while the imaginary part has a relative smallness of the order of $1 / \sqrt{\omega}$. In the intermediate frequency range $\omega \sim E$, the quantities $\operatorname{Re} M_{l^{\prime} l}^{L}$ and $\operatorname{Im} M_{l^{\prime} l}^{L}$ are of the same order of magnitude so that transitions through various intermediate states corresponding to the real part of $M_{l^{\prime} l}^{L}$ become as significant as "cascade" transi- tions. By way of an example, we give below a number of MEs $M_{l_{f} l_{i}}^{L}(E+2 \omega, E+\omega, E)$ for the energy $E=$ $0.0536=1.458 \mathrm{eV}$ and $\omega=0.0735=2.0 \mathrm{eV}$, which appear when we estimate the contribution of corrections to the photoionization cross section of the state of a hydrogen atom with $n=5, l=4$, which are quadratic in intensity and associated with virtual transitions in the continuum [68] (the results are given for the dipole interaction operator in the "form of length," which was used in [68]):

$$
\begin{gathered}
M_{35}^{4}=-(14.71+i \times 24.76), \\
M_{55}^{4}=-(3.882+i \times 0.910) \times 10^{2}, \\
M_{55}^{6}=(3.323-i \times 0.594) \times 10^{2}, \\
M_{75}^{6}=-(2.386-i \times 3.100) \times 10^{2}, \\
M_{13}^{2}=-(1.353+i \times 2.793) \times 10^{2}, \\
M_{33}^{2}=-(1.369+i \times 0.606) \times 10^{3}, \\
M_{33}^{4}=(1.141-i \times 0.346) \times 10^{3}, \\
M_{53}^{4}=-(0.819+i \times 1.270) \times 10^{3} .
\end{gathered}
$$

It can be seen that, in the case under investigation, the pole approximation (as well as the Bethe rule) is inapplicable; this situation is typical of "superthreshold" multiphoton processes, when the photon frequencies are comparable to the electron energy in the continuum. It should also be borne in mind that individual radial MEs in the total amplitude of the process "interfere" considerably (cancelled out) as a rule; this renders the requirements to their accuracy much more stringent.

Approximation (59) (1-delta approximation [14]) takes into account exactly the real part of the integrated terms in relations (38)-(40) along with the imaginary part of $M_{l^{\prime} l}^{L}$. It was established in Section 5 that this leads to correct results in the low-frequency range (for any values of the initial electron energy) in the Born region and gives a quite accurate result for two out of four MEs $M_{l^{\prime} l}^{L}$ (those whose final state has the smallest possible orbital angular momentum, see Subsection 5.2) as well as the correct form of the frequency dependence and the order of magnitude of the remaining two MEs in the high-frequency region. Consequently, this approximation is in good agreement with exact results practically for all values of the laser field frequency and electron energy (see Figs. 3 and 4). A noticeable discrepancy is observed only for small energies and for small values of momentum $l$ (the largest discrepancy is observed for the MEs in which the integrated terms do not provide a correct high-frequency asymptotic form). 

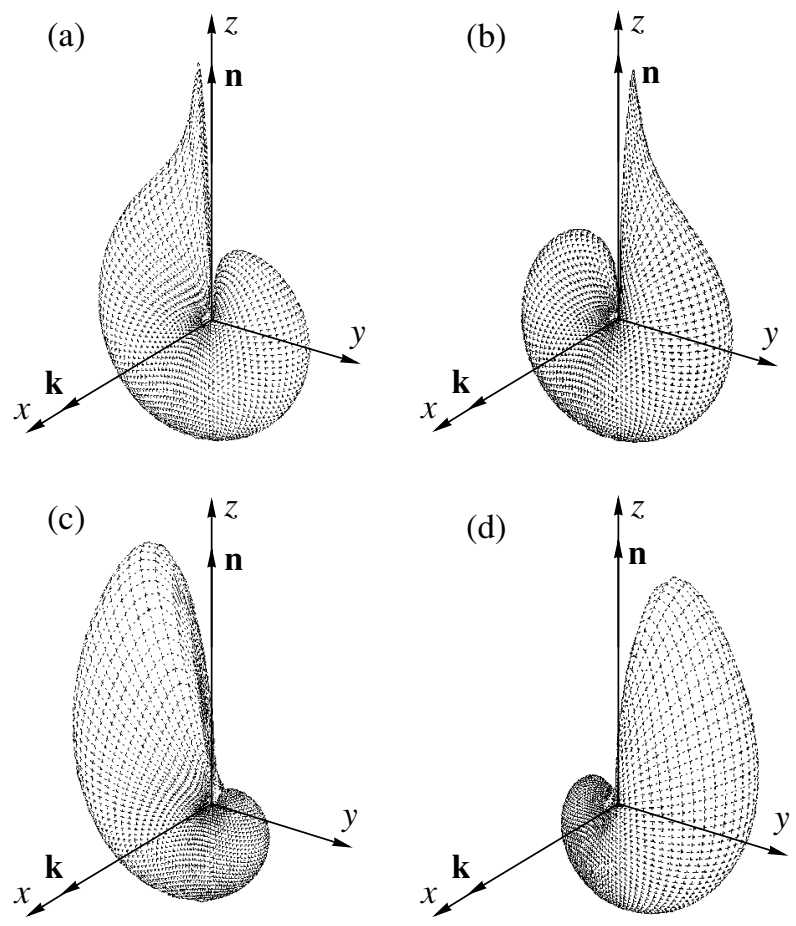

Fig. 5. Spatial distribution of electrons in $2 \mathrm{BrS}$, which are scattered (with initial energy $E=1.0$ ) by a Coulomb center in the presence of a circularly polarized wave with frequency $\omega=0.01(\mathrm{a}, \mathrm{b})$ and $\omega=0.1(\mathrm{c}, \mathrm{d})$ for $\xi=1(\mathrm{a}, \mathrm{c})$ and $\xi=-1(b, d)$. The initial electron momentum lies in the polarization plane ( $y z$ plane).

\subsection{Angular Distributions and Dichroism in Induced Bremssrahlung Processes}

It was established in Subsection 2.3 that angular distributions depend considerably not only on the initial electron energy $E$ and frequency $\omega$, but also on the polarization of photons. Figure 5 shows qualitative features of the spatial distribution of electrons scattered by a Coulomb center in the presence of a circularly polarized wave with $\xi= \pm 1$ in the case of induced $2 \mathrm{BrS}$ for two frequency values of $\omega=0.01$ and 0.1 , In accordance with the geometry presented in Fig. 2, the direction $\mathbf{k}$ of the light beam is chosen to be orthogonal to the initial momentum $\mathbf{p}=p \mathbf{n}$ of electrons with energy $E=p^{2} / 2=1.0$ au. Figure 5 clearly demonstrates the presence of CD: cross sections $d \sigma / d \Omega$ for $\xi=+1$ and $\xi=-1$ differ considerably (at the same time, they are transformed into each other upon reflection relative to the $x z$ plane; see Subsection 2.3). The CD effect decreases with frequency since the low-frequency asymptotic form (68) contains only the "regular" term. However, in the range of small scattering angles, the values of $d \sigma / d \Omega$ for $\xi= \pm 1$ differ significantly even for $\omega / E=0.01$ (see Figs. 5a and 5b and discussion in Subsection 5.1). As the value of $\theta$ decreases further, the value of $\Delta_{\mathrm{CD}}$ again becomes smaller than $f_{\text {reg }}$, which is also confirmed by numerical calculations: it can be seen
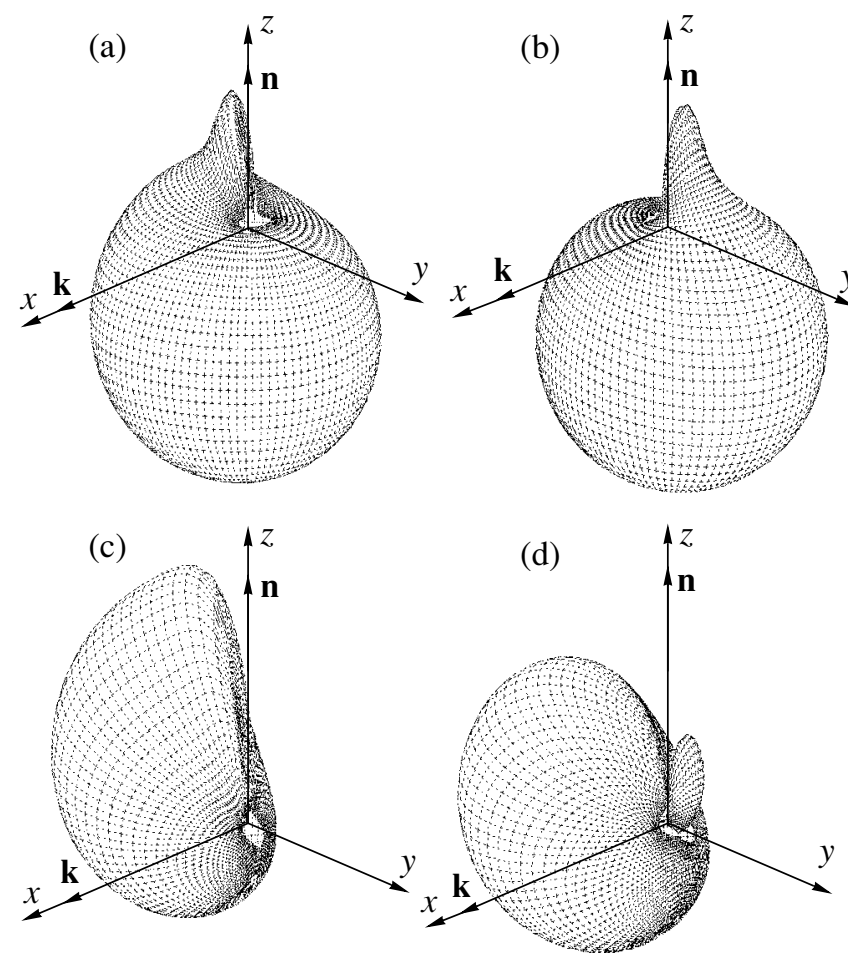

Fig. 6. The same as in Fig. 5, but in the field with elliptical polarization and frequency $\omega=0.1$ : (a) $\alpha=\pi / 2$. $\xi=1 / \sqrt{2}$; (b) $\alpha=\pi / 2, \xi=-1 / \sqrt{2}$; (c) $\alpha=\pi / 4, \xi=1 / \sqrt{2}$; (d) $\alpha=\pi / 4$, $\xi=-1 / \sqrt{2}$.

from Fig. 5 that the values of $d \sigma / d \Omega$ for $\xi=+1$ and -1 coincide for $\theta=0$.

In a field with elliptic polarization, the asymmetry in the angular distribution is caused, apart from $\mathrm{CD}$, by the effect of ED (the term with $\Delta_{\mathrm{ED}}$ in relation (24)). In this case, the cross section depends to a considerable extent on the orientation of the polarization ellipse, which is defined in Fig. 2 by the angle $\alpha$ between vector $\boldsymbol{\epsilon}$ and the $y$ axis. Figure 6 shows the angular distribution of electrons for the same values of $E$ and $\omega$ as in Figs. $5 \mathrm{c}$ and $5 \mathrm{~d}$, but for $\xi= \pm 1 / \sqrt{2}$ and for two values of angle $\alpha: \alpha=\pi / 2(\boldsymbol{\epsilon} \| \mathbf{p})$ and $\alpha=\pi / 4$ (vector $\mathbf{p}$ forms the angle $\pi / 4$ with the principal axis of the polarization ellipse). Figures $6 c$ and $6 d$ visually illustrate the reduction of symmetry upon a transition from the circular to elliptical polarization, which was considered in Subsection 2.3: for $\alpha=\pi / 4$, the results obtained for $\xi=$ $1 / \sqrt{2}$ and $\xi=-1 / \sqrt{2}$ differ qualitatively, although the $y z$ plane remains the symmetry plane as before. It should be noted that although the term $\Delta_{\mathrm{ED}}$ in general formula (24) has no smallness parameter, numerical calculations show, however, that the effect of elliptical dichroism on the asymmetry of angular distributions is considerably weaker than the effect of circular dichroism.

By way of illustration of the absolute value of twophoton cross sections and their dependences on elliptic- 

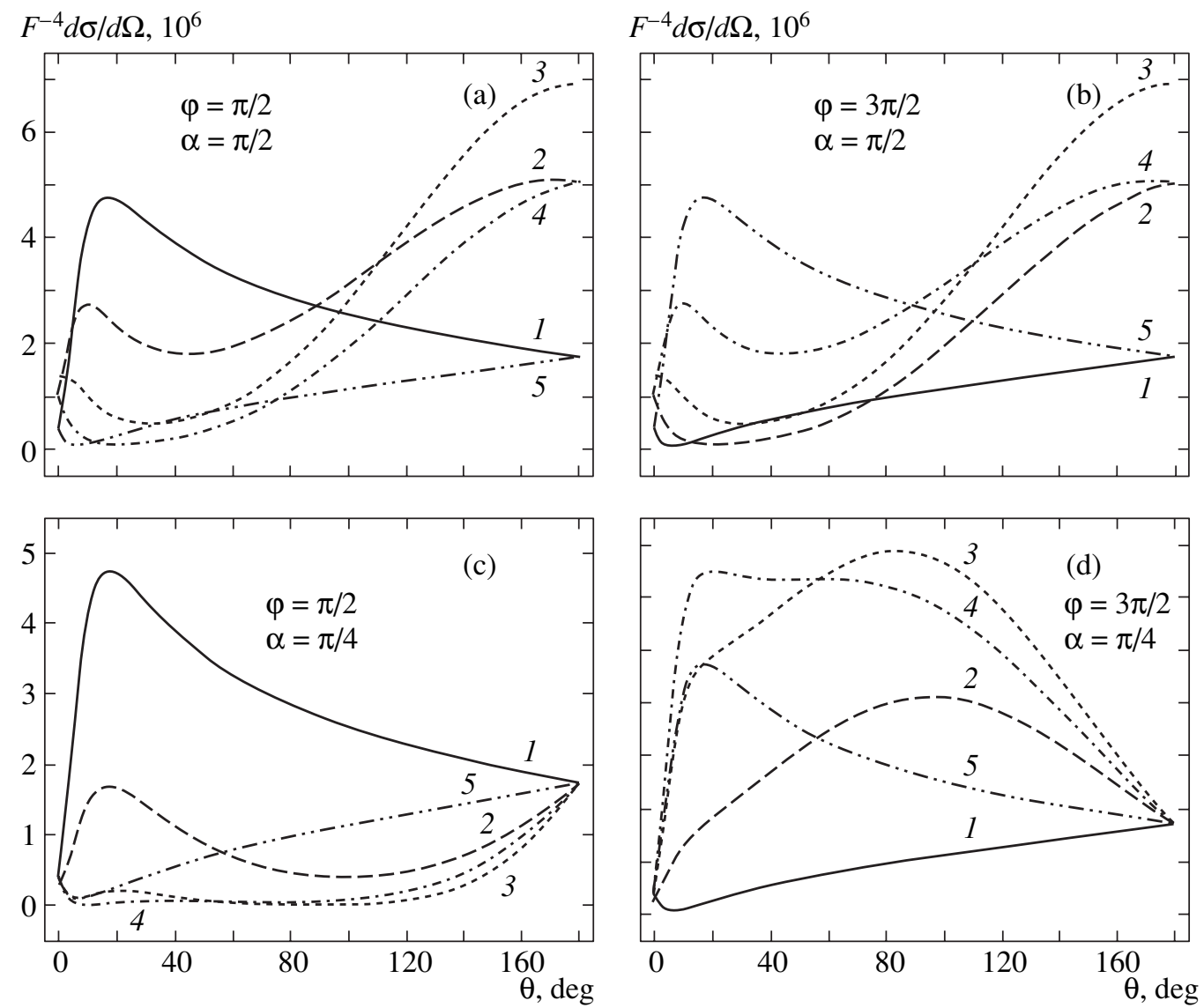

Fig. 7. Dependence of the $2 \mathrm{BrS}$ cross section for electrons with $E=1.0$ and $\omega=0.1$ on the scattering angle $\theta$ in the polarization ellipse plane: $\xi=-1(1),-1 / \sqrt{2}(2), 0(3), 1 / \sqrt{2}$ (4), and 1 (5). The values of angles $\alpha$ and $\varphi$ in the geometry of Fig. 2 are given in the figures.

ity, Fig. 7 shows the angular distributions in angle $\theta$ between $\mathbf{p}$ and $\mathbf{p}^{\prime}$ (in the plane of polarization ellipse) for induced $2 \mathrm{BrS}$ for $E=1.0, \omega / E=0.1$, and for various values of $\xi$ (the curves for double bremsstrahlung absorption have an analogous form with the substitution $\xi \longrightarrow-\xi$ ). The results are given for $\alpha=\pi / 2$ and $\alpha=\pi / 4$. In the former case $(\alpha=\pi / 2)$, for $\theta=\pi$, the cross sections are exactly identical for $\xi= \pm 1$ due to the absence of $\mathrm{CD}$ for backward scattering. At the same time, the cross sections virtually coincide in the case of elliptic polarization with $\xi= \pm 1 / \sqrt{2}$ also in view of the smallness of $\Delta_{\mathrm{ED}}$ as compared to the regular term $f_{\text {reg }}$ in Eq. (24) for $\theta=\pi$. In the latter case $(\alpha=\pi / 4)$, four curves (with $\xi= \pm 1$ and $\xi= \pm 1 / \sqrt{2}$ ) converge at one point for $\theta=\pi$ since the regular term $f_{\text {reg }}$ for these values of angles $\alpha, \theta$, and $\varphi$ is independent of polarization to a high degree of accuracy (>95\%) and makes a dominating contribution to the cross section. It can be seen that the polarization sign reversal in the range of small angles changes the cross section by almost an order of magnitude. A considerable dependence of the results on the orientation of vector $\mathbf{p}$ relative to the principal axis of the polarization ellipse and the absence of symmetry $d \sigma(\xi ; \varphi)=d \sigma(-\xi ;-\varphi)$ for $\alpha=\pi / 4$ are also worth noting.
The dependence of the two-photon absorption cross section on energy $E$ for the same values of $\xi$ and $\omega$ as in Fig. 7 is shown in Fig. 8 for two values of angle $\theta$ and orientation of $\mathbf{p}$ at right angles to the principal polarization axis $(\alpha=0)$. Dichroism effects are most significant for $\omega \sim E$, the energy dependence in this region changing qualitatively upon a variation of $\theta$.

Double bremsstrahlung absorption differs from $2 \mathrm{BrS}$ in the existence of the "critical" scattering geometry, in which the transferred momentum $\Delta \mathbf{p}=\mathbf{p}-\mathbf{p}^{\prime}$ turns out to be orthogonal to the polarization plane so that $\mathbf{e} \cdot \Delta \mathbf{p}=0$. In this case, the Born approximation (81) and the low-frequency asymptotic form (64) give zero value for the cross section, for which the value of $d \sigma / d \Omega$ is finite when the scattering potential is accurately taken into account. Figure 9 shows the dependence of the double bremsstrahlung absorption cross section on angle $\varphi$ (see Fig. 2) in the "critical" region. For energy $E=1.0$ and $\omega=1 / 6$ (Fig. 9a), the BunkinFedorov formula (81) describes the cross section quite satisfactorily (although the Born parameters are not very small in this region: $\alpha \approx 0.7$ and $\alpha^{\prime} \approx 0.6$ ) except in the small angular region near the "critical" point $\theta=$ $\pi / 6, \varphi=0$ at which the Born results vanishes, and the exact cross section has a clearly manifested minimum. 

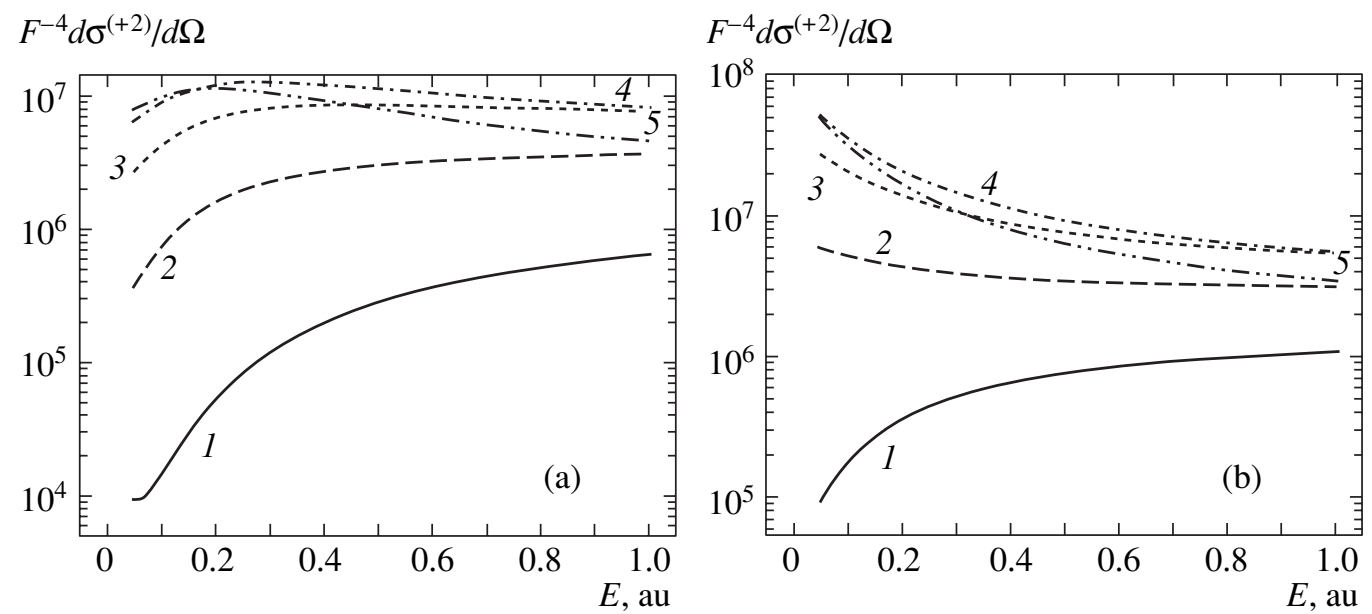

Fig. 8. Dependence of the two-photon absorption cross section on energy $E$ for $\omega=0.1, \alpha=0, \varphi=\pi / 2$ and for various $\xi$ (notation of the curves is the same as in Fig. 7) (a) $\theta=\pi / 6$; (b) $\theta=\pi / 3$.
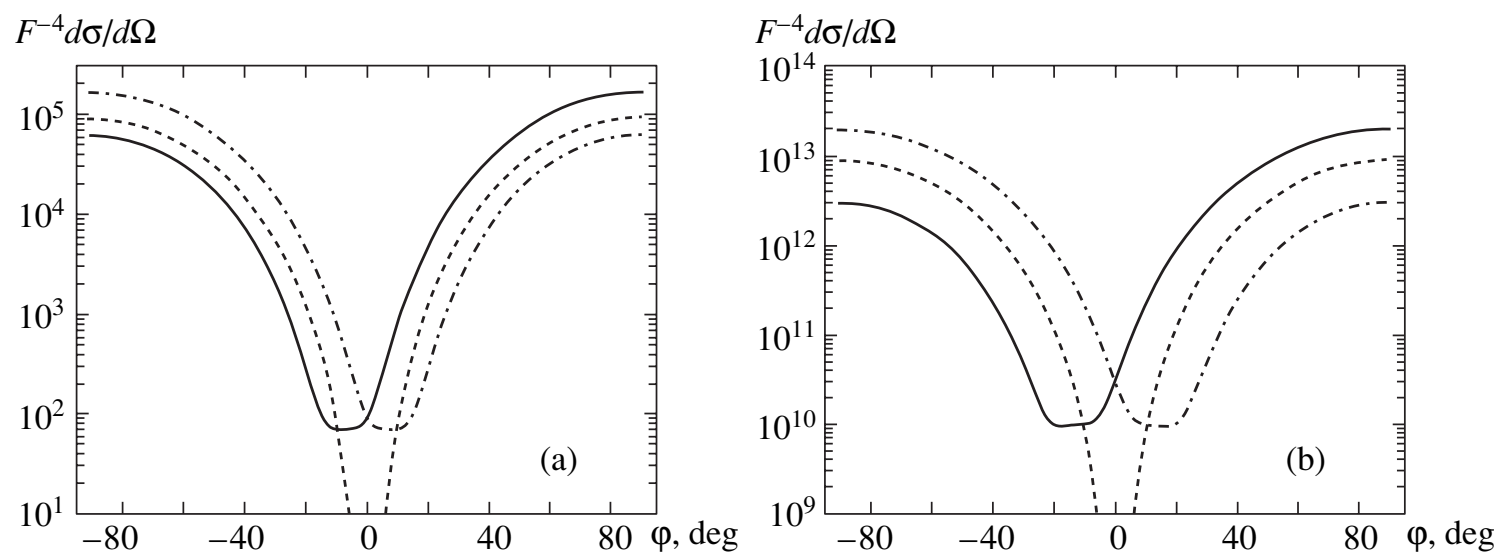

Fig. 9. Dependence of the double bremsstrahlung absorption cross section on angle $\varphi$ for $\theta=\pi / 6$ (solid curves correspond to $\xi=$ $1 / \sqrt{2}$ and dot-and-dash curves to $\xi=-1 / \sqrt{2}), E=1.0, \omega=1 / 6(($ a), the dashed curve corresponds to the Born approximation (81)) and $E=0.1, \omega=1 / 60$ ((b), the dashed curve corresponds to the low-frequency asymptotic form (64)).
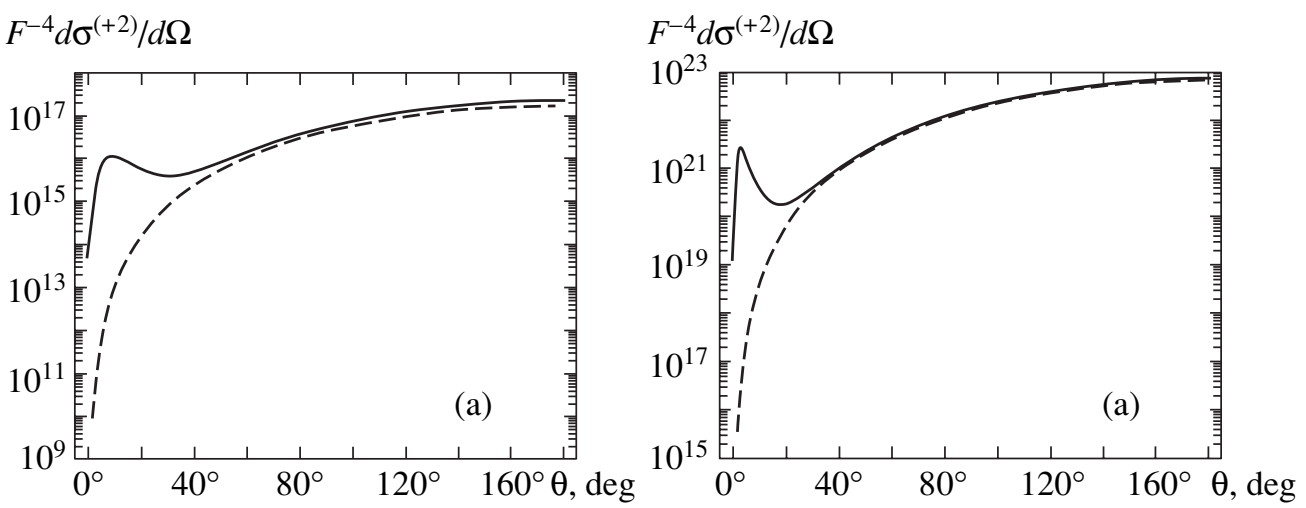

Fig. 10. Double bremsstrahlung absorption cross section for electrons with energy $E=0.1$ by a Coulomb center in the field of a linearly polarized wave with frequency $\omega=0.005$ (a) and 0.001 (b). The initial electron momentum is directed along the polarization vector of the wave; $\theta$ is the scattering angle. Solid curves correspond to the exact result and the dashed curves to the low-frequency approximation (64). 

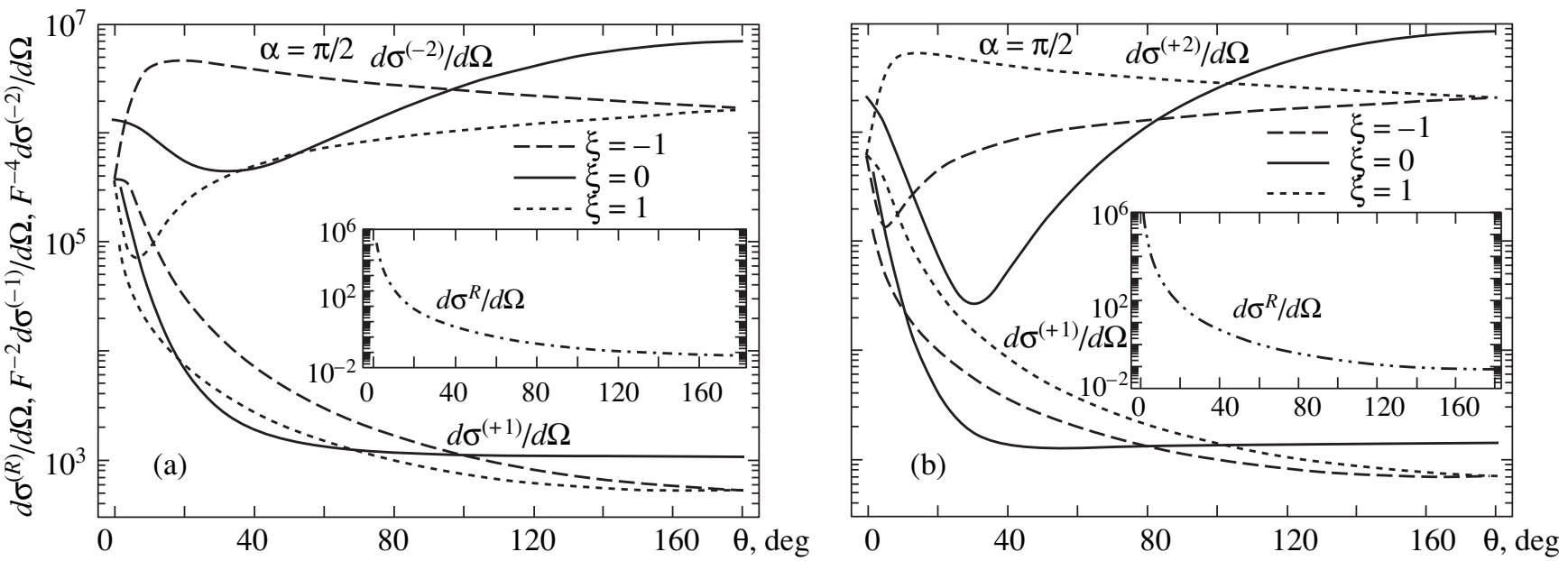

Fig. 11. Scattering cross sections for electrons with energy $E=1.0$ in the fields of linearly $(\xi=0)$ and circularly $(\xi= \pm 1)$ polarized waves with frequency $\omega=0.1$, The momentum of a scattered electron lies in the polarization plane; $\varphi=\pi / 2$. The quantities $d \sigma^{(n)} / d \Omega$ with $n=-1,-2$ and $n=+1,+2$ correspond to (a) emission and (b) absorption of one or two photons. The insets show the cross section $d \sigma^{(R)} / d \Omega$ of Rutherford scattering in zero field.

At the same time, the low-frequency asymptotic form (64) for $E=0.1$ and $\omega=1 / 60$ (Fig. 9b) leads to a much worse quantitative agreement with exact results. Another region of "critical" parameters, in which the low-frequency Kroll-Watson asymptotic form leads to a strong discrepancy with experimental values and which has been discussed actively during recent years (see references in [32]) is the low-energy scattering (with energy $E$ on the order of several electronvolts) via small angles in a linearly polarized field with the initial momentum $\mathbf{p}$ along the direction of polarization. Figure 10 shows the double bremsstrahlung absorption cross sections for $E=0.1$ and $\omega / E=0.01$ and 0.05 in this geometry. It can be seen that the difference between exact and approximate results for small angles can be as large as five to six orders of magnitude, although an increase in $\theta$ leads to nearly complete agreement (the

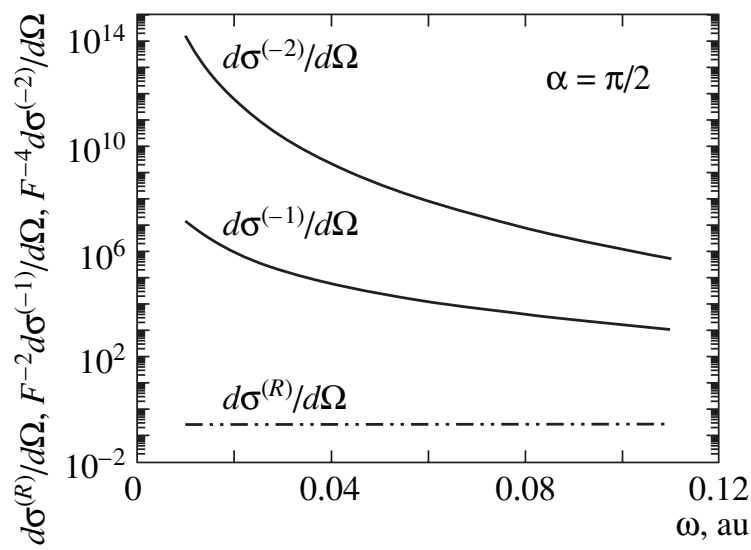

Fig. 12. Frequency dependence of the single-photon $\left(d \sigma^{(-1)} / d \Omega\right)$ and double bremsstrahlung $\left(d \sigma^{(-2)} / d \Omega\right)$ emission of electrons with energy $E=1.0$ by a Coulomb center in the field of a circularly polarized wave with $\xi=1$ in the geometry of Fig. 2 with $\theta=\varphi=\pi / 2 ; d \sigma^{(R)} / d \Omega$ is the Rutherford scattering cross section. results for $2 \mathrm{BrS}$ are qualitatively the same as in Fig. 10, but the difference for small angles $\theta$ in this case does not exceed one or two orders of magnitude). Thus, the problem of simple approximations of bremsstrahlung cross sections for describing small-angle scattering at low energies (when the Born approximation is inapplicable) requires special analysis.

Figure 11 illustrates the relation between the cross sections of one- and two-photon scattering as functions of the laser radiation parameters and scattering angle. The cross sections of conventional Rutherford scattering $\left(d \sigma^{(R)} / d \Omega\right)$ and one- and two-photon absorptions $\left(d \sigma^{(n)} / d \Omega\right.$ with $\left.n= \pm 1, \pm 2\right)$ are given as functions of angle $\theta$ in the polarization plane (analytic expressions for factors $Q$ in the amplitude of single-photon processes (3) and (4) are given in [4] for the Coulomb potential). It can be seen that the cross sections $\left(d \sigma^{(n)} / d \Omega\right.$ are more sensitive to a change in the ellipticity for angles $\theta<\pi / 2$; in both cases, for small angles, the cross sections have maximal values for circular polarization, while scattering via angles $\theta>\pi / 2$ is more effective in a field with linear polarization. In contrast to the sharp angular dependence of the Rutherford cross section $d \sigma^{(R)} / d \Omega$ for small angles $\theta$, the cross sections of bremsstrahlung processes depend on $\theta$ more smoothly, although single-photon cross sections also increase by more than two orders of magnitude for small angles, while two-photon processes exhibit a nonmonotonic dependence. Dichroism effects are the most significant for small angles, although the difference in the cross sections for scattering at right angles attains $100 \%$ upon the sign reversal of $\xi$. For a fixed initial electron energy, the absolute value of bremsstrahlung cross sections increases sharply with decreasing frequency (Fig. 12); in this case, the frequency dependences are successfully approximated by the expression

$$
d \sigma^{( \pm n)}(\omega) / d \Omega \sim 1 / \omega^{4 n} .
$$


It should be noted in conclusion that the above results make it possible to qualitatively estimate the limits of applicability of perturbation theory in the laser field for describing bremsstrahlung processes. For example, in the range of small angles, for $E \approx 1$ and $\omega \approx$ 0.1 , a typical ratio of two-photon to one-photon cross sections amounts to $\left(10^{3}-10^{4}\right) F^{2}$ and attains unity (when perturbation theory becomes obviously inapplicable) for an intensity $I_{\text {cr }} \sim\left(10^{12}-10^{13}\right) \mathrm{W} / \mathrm{cm}^{2}$, which is much smaller than the intraatomic intensity $I_{0}=3.51 \times$ $10^{16} \mathrm{~W} / \mathrm{cm}^{2}$. The value of $I_{\text {cr }}$ decreases rapidly with frequency (in proportion to $\omega^{4}$ ) so that the effective parameter of perturbation theory in the optical frequency range is $F / \omega^{2}$ (or the ratio of the amplitude of classical oscillations of an electron in a laser field to the Born radius). For small $\theta$, the ratio $d \sigma^{( \pm 2)}(\omega) / d \sigma^{( \pm 1)}$ attains unity only in ultrastrong fields with $I_{\mathrm{cr}} \sim I_{0}$ (see Fig. 11), while the dominating contribution to the cross section of scattering via small angles in weaker fields comes from single-photon processes.

\section{ACKNOWLEDGMENTS}

This study was partly financed by the CRDF and the Ministry of Education of the Russian Federation (grant no. VZ-010-0) as well as by the Russian Foundation for Basic Research (project no. 00-02-17843), the Competition Center of the Education Ministry of the Russian Federation (grant no. E00-3.2-515), and the National Science Foundation of the USA (AFS, grant no. PHY-0070980).

\section{APPENDIX A}

Using the recurrence relations for the degenerate hypergeometric function $\Phi(\alpha, \beta ; x)$, we can present the action of the dipole operators (22) on the Coulomb wave function $R_{E l}(r)$ of the continuous spectrum in the form

$$
\begin{gathered}
D(l+1, l) R_{E l}(r)=-p \frac{l+1-i a}{|l+1-i a|} R_{E l+1}(r) \\
\quad-\frac{2 Z C_{E l}}{(2 l+3) !}(2 p r)^{l} \exp (-i p r) \\
\times[(l+2+i a) \Phi(l+3+i a, 2 l+4 ; 2 i p r) \\
+(l+1-i a) \Phi(l+2+i a, 2 l+4 ; 2 i p r)], \\
D(l+1, l+2) R_{E l+2}(r)=p \frac{|l+2-i a|}{l+2-i a} R_{E l+1}(r) \\
-\frac{2 Z C_{E l+2}}{(2 l+3) !(l+2-i a)}(2 p r)^{l} \exp (-i p r) \\
\quad \times[\Phi(l+3+i a, 2 l+4 ; 2 i p r) \\
-\Phi(l+2+i a, 2 l+4 ; 2 i p r)] .
\end{gathered}
$$

Since the first terms on the right-hand sides of relations (A.1) and (A.2) are proportional to $R_{E l+1}(r)$, the corresponding terms in the MEs $M_{l^{\prime} l}^{L}$ in relations (36) can be expressed in terms of MEs (37) in accordance with relations (38)-(40). The values of $d_{l^{\prime} l}$ can easily be calculated using relations (A.1) and (A.2), which gives formulas (41) for $d_{l+1 l}$ and $d_{l+1 l+2}$, in which the integral

$$
\begin{gathered}
I^{m^{\prime} m}\left(E^{\prime}, E\right)=\int_{0}^{\infty} d r r^{2 l+3} \exp \left[-i\left(p^{\prime}+p\right) r-\varepsilon r\right] \\
\times \Phi\left(l+3-m^{\prime}+i a^{\prime}, 2 l+4 ; 2 i p^{\prime} r\right) \\
\quad \times \Phi\left(l+3-m+i a, 2 l+4 ; 2 i p^{\prime} r\right)
\end{gathered}
$$

is a tabular integral [56], which can be reduced to the functions ${ }_{2} F_{1}$ (see relation (42)).

The double integral $J^{m^{\prime} m}$ in relations (38)-(40) contains the radial Coulomb Green's function $g_{l}\left(r, r^{\prime}, \mathscr{E}\right)$ :

$$
\begin{aligned}
& J^{m^{\prime} m}=\int_{0}^{\infty} d r^{\prime} d r\left(r^{\prime} r\right)^{l+2} \exp \left[-i p^{\prime} r^{\prime}-i p r-\varepsilon r\right] \\
& \times \Phi\left(l+3-m^{\prime}+i a^{\prime}, 2 l+4 ; 2 i p^{\prime} r\right) \\
& \times g_{l+1}\left(\mathscr{E}, r, r^{\prime}\right) \Phi(l+3-m+i a, 2 l+4 ; 2 i p r) .
\end{aligned}
$$

In order to evaluate this integral, it is convenient to use the integral representation of $g_{L}[69]$ :

$$
\begin{aligned}
& g_{L}\left(r, r^{\prime}, \mathscr{E}\right)=\frac{2}{\sqrt{r r^{\prime}}} \int_{0}^{1} \frac{d t}{1-t} t^{-(1 / 2)-Z v} \\
& \times \exp \left[-\frac{r+r^{\prime}}{v} \frac{1+t}{1-t}\right] I_{2 L+1}\left(\frac{4 \sqrt{r r^{\prime} t}}{v(1-t)}\right),
\end{aligned}
$$

where $I_{k}(t)$ is a modified Bessel's functions $v=$ $1 / \sqrt{-2 \mathscr{E}}$. It should be noted that integrals (A.3) and (A.4) with oscillating functions contain regularizing factors $\exp (-\varepsilon r)$ with $\varepsilon \longrightarrow+0$. Substituting expression (A.5) into integral (A.4) and using the integral representation of the function $\Phi(\alpha, \beta, x)$ [56], we obtain

$$
\begin{gathered}
=\frac{J^{m^{\prime} m}\left(E^{\prime}, E, \mathscr{E}\right)}{\Gamma(l+1+m+i a) \Gamma(l+3-m-i a)} \\
\times \int_{0}^{1} d t \frac{t^{-Z v-1 / 2}}{1-t} \int_{0}^{1} d u u^{l+m+i a}(1-u)^{l+2-m^{\prime}-i a} \\
\times \int_{0}^{\infty} d r^{\prime} r^{l+3 / 2} \exp \left[-\left(\frac{1}{v} \frac{1+t}{1-t}+i p^{\prime}\right) r^{\prime}\right] \\
\times \Phi\left(l+1+m^{\prime}+i a^{\prime}, 2 l+4 ; 2 i p^{\prime} r^{\prime}\right) \int_{0}^{\infty} d r r^{l+3 / 2} \\
\times \exp \left[-\left(i p+\frac{1}{v} \frac{1+t}{1-t}-2 i p u\right) r\right] I_{2 l+3}\left(\frac{4 \sqrt{r r^{\prime} t}}{v(1-t)}\right) .
\end{gathered}
$$


The special relation between the index of Bessel's function $I_{k}$, the parameter $\beta$ in $\Phi(\alpha, \beta ; x)$, and the exponents $r$ and $r^{\prime}$ in integral (A.6), which was obtained using transformations (A.1) and (A.2), makes it possible to express the integrals with respect to $r$ and $r^{\prime}$ in terms of elementary functions [63]. As a result, the integral with respect to $u$ in relation (A.6) gives a definition of the hypergeometric function of two variables (Appel's function),

$$
F_{1}\left(l+1+m+i a ; l+3-m^{\prime}-i a^{\prime}, l+1+m^{\prime}+i a^{\prime} ; 2 l+4 ; x, y\right),
$$

which can be reduced, for the above values of parameters, to ${ }_{2} F_{1}$ in accordance with the well-known reduction formulas [56], and $J^{m ' m}$ assumes the final form (43).

\section{APPENDIX B}

Analytic continuation of formulas (38)-(40) in variables $p$ and $p^{\prime}$ to the range of negative energies, $p \longrightarrow$ $i Z / n$ and $p^{\prime} \longrightarrow i Z / n^{\prime}$, makes it possible to obtain the
MEs of two-photon transitions between the states $|n l\rangle$ and $\left|n^{\prime} l^{\prime}\right\rangle$ of the discrete spectrum:

$$
M_{l^{\prime} l}^{L}\left(n^{\prime}, n, \mathscr{E}\right)=\left\langle n^{\prime} l^{\prime}\left|D\left(l^{\prime}, L\right) g_{L}(\mathscr{E}) D(L, l)\right| n l\right\rangle .
$$

As a result of such a substitution for $p$ and $p^{\prime}$, the parameters of the functions ${ }_{2} F_{1}$ and the exponents in the denominator in integral (43) become integral:

$$
J^{m^{\prime} m}\left(n^{\prime}, n, \mathscr{E}\right) \propto \int_{0}^{1} d t \frac{t^{l+1-Z v}{ }_{2} F_{1}\left(l+1+m^{\prime}-n^{\prime}, l+1+m-n, 2 l+4 ; \lambda\right)}{(1-t / y)^{l+1+m-n}(1-y t)^{l+1+m^{\prime}-n^{\prime}}\left(1-y^{\prime} t\right)^{2-m^{\prime}-m+n^{\prime}+n}},
$$

where

$$
\begin{gathered}
y=\frac{(\alpha-v)\left(\alpha^{\prime}+v\right)}{(\alpha+v)\left(\alpha^{\prime}-v\right)}, \quad y^{\prime}=\frac{(\alpha-v)\left(\alpha^{\prime}-v\right)}{(\alpha+v)\left(\alpha^{\prime}+v\right)}, \\
\lambda=\frac{16 \alpha \alpha^{\prime} v^{2}}{\left(\alpha^{2}-v^{2}\right)\left(\alpha^{\prime 2}-v^{2}\right)} \frac{t}{(1-y t)(1-t / y)}, \\
\alpha=n / Z, \quad \alpha^{\prime}=n^{\prime} / Z .
\end{gathered}
$$

The integral in formula (B.2) can be expressed in terms of the known functions by transforming the integrand with the help of the relation (see formula (2.5.2.12) in [56])

$$
\begin{gathered}
\frac{(1-y t)^{n^{\prime}-l-m^{\prime}-1}(1-t / y)^{n-l-m-1}}{\left(1-y^{\prime} t\right)^{n+n^{\prime}+2-m-m^{\prime}}} \\
\times{ }_{2} F_{1}\left(l+1+m^{\prime}-n^{\prime}, l+1+m-n, 2 l+4 ; \lambda\right)
\end{gathered}
$$

$$
\begin{gathered}
=\sum_{k=0}^{\infty} \frac{(2 l+4)_{k}}{k !}\left(y^{\prime} t\right)_{2}^{k} F_{1}(l+1+m-n,-k ; 2 l+4 ; z) \\
\quad \times{ }_{2} F_{1}\left(l+1+m^{\prime}-n^{\prime},-k ; 2 l+4 ; z^{\prime}\right),
\end{gathered}
$$

where $z=-4 \alpha v /(\alpha-v)^{2}, z^{\prime}=-4 \alpha^{\prime} v /\left(\alpha^{\prime}-v\right)^{2}$. After this, the integral in formula (B.2) can be evaluated easily and has the form identical for formula (13) from [49]. Using the results obtained in [49], we can write $J^{m^{\prime} m}$ in terms of the hypergeometric polynomials ${ }_{2} F_{1}(-k, b ; c ; x)$ and Appel's function $F_{1}(a ;-k, k+2 l+2 ; d ; x, y)$ of special form, which can be expressions through the sum of $k+$ 1 complete functions ${ }_{2} F_{1}$ or (in the case of negative integral $a$ ) through the hypergeometric polynomial of two variables:

$$
\begin{gathered}
J^{m^{\prime} m}=2^{-2 l-4} \Gamma^{2}(2 l+4)\left(\alpha \alpha^{\prime}\right)^{l+2} \\
\times g_{n-l-1-m, n^{\prime}-l-1-m^{\prime}}^{l+1}\left(\nu ; \alpha, \alpha^{\prime}\right),
\end{gathered}
$$

where

$$
\begin{gathered}
g_{k k^{\prime}}^{l}\left(v ; \alpha, \alpha^{\prime}\right)=v \frac{\left(4 v \sqrt{\alpha \alpha^{\prime}}\right)^{2 l+2}}{\Gamma(2 l+2)} \frac{(\alpha-v)^{k}}{(\alpha+v)^{k+2 l+2}} \frac{\left(\alpha^{\prime}-v\right)^{k^{\prime}}}{\left(\alpha^{\prime}+v\right)^{k^{\prime}+2 l+2}}\left[\frac{{ }_{2} F_{1}(-k, l+1-\eta ; 2 l+2 ; z)}{l+1-\eta}\right. \\
\quad \times F_{1}\left(l+1-\eta ;-k^{\prime} ; k^{\prime}+2 l+2 ; l+2-\eta ; y, y^{\prime}\right) \\
\left.+\sum_{p=1}^{k} C_{k}^{p}(-z)^{p} \frac{{ }_{2} F_{1}(-k+p, l+1-\eta+p ; 2 l+2+p ; z)}{(2 l+2)_{p}} \Phi_{p}\left(k^{\prime}, l, \eta ; y, y^{\prime}\right)\right],
\end{gathered}
$$




$$
\begin{gathered}
\Phi_{p}\left(k^{\prime}, l, \eta ; y, y^{\prime}\right)=-\frac{(l+2+\eta-p)_{p-1}(1-y)^{k^{\prime}}}{\left(1-y^{\prime}\right)^{k^{\prime}+2 l+2}} \\
\times F_{1}\left(-p+1 ;-k^{\prime}, k^{\prime}+2 l+2 ; l+2+\eta-p ; 1 /(1-y), 1 /\left(1-y^{\prime}\right)\right),
\end{gathered}
$$

$C_{k}^{p}$ is the binomial coefficient, and $\eta=Z v$.

In order to transform $J^{m^{\prime m}}$ to (B.3), (B.4), it is sufficient that only one of the two numbers $\left(n\right.$ or $\left.n^{\prime}\right)$ in formula (B.2) be an integer (this number will determine the upper limit $k$ of the sum over $p$ in formulas (B.4)). Consequently, the amplitudes of free-bound (but not free-free) transitions can also be presented in closed form. Explicit expressions for all $M_{l^{\prime} l}^{L}\left(n^{\prime}, n, \mathscr{E}\right)$ in formula (B.1), which are allowed by the dipole selection rules, in the form of linear combinations of $g_{k k^{\prime}}^{l}$, as well as analogous expressions for the MEs of bound-free transitions (two-photon Gordon's formula), are given in [49].

\section{REFERENCES}

1. A. Sommerfeld, Ann. Phys. (Leipzig) 11, 257 (1931); A. Sommerfeld, Atomic Structure and Spectral Lines (Methuen, London, 1934; Gostekhizdat, Moscow, 1956), Vol. 2.

2. L. Bess, Phys. Rev. 77, 550 (1950); A. Nordsieck, Phys. Rev. 93, 785 (1954).

3. V. B. Berestetskii, E. M. Lifshitz, and L. P Pitaevskii, Course of Theoretical Physics, Vol. 4: Quantum Electrodynamics (Nauka, Moscow, 1980; Pergamon, New York, 1982).

4. N. L. Manakov, S. I. Marmo, and V. V. Volovich, Phys. Lett. A 27A, 42 (1995); J. Electron Spectrosc. Relat. Phenom. 79, 327 (1996).

5. L. C. Biedenharn, Phys. Rev. 102, 262 (1956).

6. W. Heitler and L. Nordheim, Physica (Amsterdam) 1, 1059 (1934).

7. J. C. Altman and C. A. Quarles, Phys. Rev. A 31, 2744 (1985).

8. D. L. Kahler, J. Liu, and C. A. Quarles, Phys. Rev. Lett. 68, 1690 (1992); Phys. Rev. A 47, 2819 (1993); J. Liu and C. A. Quarles, Phys. Rev. A 47, R3479 (1993).

9. R. Hippler, Phys. Rev. Lett. 66, 2197 (1991); R. Hippler and H. Schneider, Nucl. Instrum. Methods Phys. Res. B 87, 268 (1994).

10. A. I. Smirnov, Yad. Fiz. 25, 1030 (1977) [Sov. J. Nucl. Phys. 25, 548 (1977)].

11. M. Gavrila, A. Maquet, and V. Veniard, Phys. Rev. A 32, 2537 (1985); V. Veniard, M. Gavrila, and A. Maquet, Phys. Rev. A 35, 448 (1987); M. Gavrila, A. Maquet, and V. Veniard, Phys. Rev. A 42, 236 (1990).

12. V. Florescu and V. Djamo, Phys. Lett. A 119, 73 (1986); M. Dondera and V. Florescu, Phys. Rev. A 48, 4267 (1993).

13. N. L. Manakov, S. I. Marmo, and A. G. Faĭnshteĭn, Zh. Éksp. Teor. Fiz. 108, 1569 (1995) [JETP 81, 860 (1995)].
14. A. V. Korol, J. Phys. B 28, 3873 (1995).

15. A. V. Korol, J. Phys. B 29, 3257 (1996).

16. T. A. Fedorova, A. V. Korol, and I. A. Solovjev, J. Phys. B 33, 5007 (2000).

17. A. V. Korol, J. Phys. B 30, 413 (1997).

18. M. Dondera and V. Florescu, Phys. Rev. A 58, 2016 (1998).

19. A. V. Korol, J. Phys. B 27, 155 (1994).

20. G. Kracke, J. S. Briggs, A. Dubois, et al., J. Phys. B 27, 3241 (1994).

21. D. Andrick and L. Langhaus, J. Phys. B 9, L459 (1976).

22. A. Weingartshofer, J. K. Holmes, G. Caudle, et al., Phys. Rev. Lett. 39, 269 (1977).

23. B. Wallbank and J. K. Holmes, Phys. Rev. A 48, R2515 (1993); J. Phys. B 27, 1221 (1994); 27, 5405 (1994); 29, 5881 (1996).

24. N. J. Mason, Rep. Prog. Phys. 56, 1275 (1993).

25. F. V. Bunkin and M. V. Fedorov, Zh. Éksp. Teor. Fiz. 49, 1569 (1965) [Sov. Phys. JETP 22, 1071 (1966)].

26. F. V. Bunkin, A. E. Kazakov, and M. V. Fedorov, Usp. Fiz. Nauk 107, 559 (1972) [Sov. Phys. Usp. 15, 416 (1972)].

27. N. M. Kroll and K. M. Watson, Phys. Rev. A 8, 804 (1973).

28. M. H. Mittleman, Phys. Rev. A 19, 134 (1979); 20, 1965 (1979); 21, 79 (1980).

29. L. Rosenberg, Phys. Rev. A 23, 2283 (1981).

30. S. Geltman, Phys. Rev. A 53, 3473 (1996); 55, 3755 (1997).

31. L. B. Madsen and K. Taulbjerg, J. Phys. B 31, 4701 (1998).

32. N. J. Kylstra and C. J. Joachain, Phys. Rev. A 58, R26 (1998); 60, 2255 (1999).

33. L. W. Garland, A. Jaroń, J. Z. Kamiń ski, and R. M. Potvliege, J. Phys. B 35, 2861 (2002).

34. I. Ya. Berson, Zh. Éksp. Teor. Fiz. 80, 1727 (1981) [Sov. Phys. JETP 53, 891 (1981)].

35. F. Ehlotzky, A. Jaroń, and J. Z. Kamiński, Phys. Rep. 297, 63 (1998).

36. A. G. Fainshtein, N. L. Manakov, and S. I. Marmo, Phys. Lett. A 195, 358 (1994).

37. R. V. Karapetyan and M. V. Fedorov, Zh. Éksp. Teor. Fiz. 75, 816 (1978) [Sov. Phys. JETP 48, 412 (1978)].

38. R. Daniele and E. Fiordilino, Nuovo Cimento D 18, 547 (1996).

39. A. Florescu and V. Florescu, Phys. Rev. A 61, 033406 (2000).

40. V. P. Kraĭnov and S. P. Roshchupkin, Zh. Éksp. Teor. Fiz. 84, 1302 (1983) [Sov. Phys. JETP 57, 754 (1983)].

41. A. Florescu and V. Florescu, Phys. Lett. A 226, 280 (1997).

42. M. H. Mittleman, J. Phys. B 26, 2709 (1993). 
43. P. D. Fainstein and A. Maquet, J. Phys. B 27, 5563 (1994).

44. D. Khalil, O. E. Akramine, A. Makhoute, et al., J. Phys. B 31, 1115 (1998); O. E. Akramine, A. Makhoute, D. Khalil, et al., J. Phys. B 32, 2783 (1999).

45. N. L. Manakov, S. I. Marmo, and A. V. Meremianin, J. Phys. B 29, 2711 (1996).

46. A. A. Balakin and G. M. Fraĭman, Zh. Éksp. Teor. Fiz. 120, 797 (2001) [JETP 93, 695 (2001)].

47. L. P. Rapoport and A. S. Kornev, Zh. Éksp. Teor. Fiz. 116, 1241 (1999) [JETP 89, 664 (1999)]; L. P. Rappoport and A. S. Kornev, J. Phys. B 33, 87 (2000).

48. A. Cionga, F. Ehlotzky, and G. Zloh, Phys. Rev. A 62, 063406 (2000); J. Phys. B 33, 4939 (2000).

49. A. A. Krylovetskiǔ, N. L. Manakov, and S. I. Marmo, Zh. Éksp. Teor. Fiz. 119, 45 (2001) [JETP 92, 37 (2001)].

50. D. A. Varshalovich, A. N. Moskalev, and V. K. Khersonskii, Quantum Theory of Angular Momentum (Nauka, Leningrad, 1975; World Sci., Singapore, 1988).

51. N. L. Manakov, A. V. Meremianin, and A. F. Starace, Phys. Rev. A 57, 3233 (1998).

52. J. S. Briggs and V. Schmidt, J. Phys. B 33, R1 (2000).

53. N. L. Manakov, A. Maquet, S. I. Marmo, et al., J. Phys. B 32, 3747 (1999).

54. N. L. Manakov and A. V. Merem'yanin, Zh. Éksp. Teor. Fiz. 112, 1984 (1997) [JETP 84, 1080 (1997)].

55. H. A. Bethe and E. E. Salpeter, Quantum Mechanics of One- and Two-Electron Atoms (Academic, New York, 1957; Fizmatgiz, Moscow, 1960).

56. Heigher Transcendental Functions (Bateman Manuscript Project), Ed. by A. Erdelyi (McGraw-Hill, New York, 1953; Nauka, Moscow, 1974), Vol. 1.

57. L. D. Landau and E. M. Lifshitz, Course of Theoretical Physics, Vol. 3: Quantum Mechanics: Non-Relativistic
Theory (Nauka, Moscow, 1974; Pergamon, New York, 1977).

58. L. C. Biedenharn, J. L. McHale, and R. M. Thaler, Phys. Rev. 100, 376 (1955).

59. N. L. Manakov, M. A. Preobrazhenskiǔ, L. P. Rapoport, and A. G. Faŭnshteĭn, Zh. Éksp. Teor. Fiz. 75, 1243 (1978) [Sov. Phys. JETP 48, 626 (1978)]; N. C. Sil, M. A. Crees, and M. J. Seaton, J. Phys. B 17, 1 (1984); B. Gao and A. F. Starace, Comput. Phys. 6, 70 (1987).

60. J. L. Madajczyk and M. Trippenbach, J. Phys. A 22, 2369 (1989).

61. L. Pan, J. Mod. Opt. 36, 877 (1989); V. Veniard and B. Piraux, Phys. Rev. A 41, 4019 (1990); A. V. Korol, J. Phys. B 27, L103 (1994); T. Mercouris, Y. Komninos, S. Dionissopoulou, and C. A. Nicolaides, J. Phys. B 29, L13 (1996).

62. N. L. Manakov, S. I. Marmo, and A. V. Shaposhnikov, in Atoms and Molecules in Strong Field of Laser Radiation, Ed. by F. V. Bunkin and I. I. Tugov (Wiley-Nauka, Moscow, 1992), p. 87.

63. I. S. Gradshteyn and I. M. Ryzhik, Table of Integrals, Series, and Products (Nauka, Moscow, 1971; Academic, New York, 1980).

64. S. D. Oh and R. H. Pratt, Phys. Rev. A 45, 1583 (1992).

65. C. D. Shaffer, R. H. Pratt, and S. D. Oh, Phys. Rev. A 57, 227 (1998).

66. Z. Deng and J. H. Eberly, Phys. Rev. Lett. 53, 1810 (1984); J. Opt. Soc. Am. B 2, 486 (1985).

67. M. Trippenbach, K. Rzazewski, M. V. Fedorov, and A. E. Kazakov, J. Phys. B 22, 1193 (1989).

68. O. V. Tikhonova, A. M. Popov, and M. V. Fedorov, Phys. Rev. A 65, 053404 (2002).

69. L. Hostler, J. Math. Phys. 5, 591 (1964).

Translated by N. Wadhwa 